\title{
Mitochondrial PKM2 regulates oxidative stress-induced apoptosis by stabilizing $\mathrm{Bcl} 2$
}

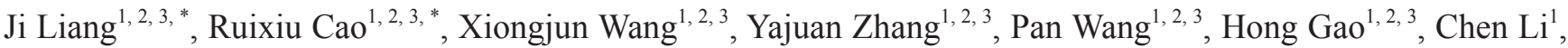
Fan Yang ${ }^{4}$, Rong Zeng ${ }^{1}$, Ping Wei ${ }^{5}$, Dawei $\mathrm{Li}^{6}$, Wenfeng $\mathrm{Li}^{7}$, Weiwei Yang ${ }^{1,2,3}$

${ }^{I}$ CAS Key Laboratory of Systems Biology, CAS Center for Excellence in Molecular Cell Science, Institute of Biochemistry and Cell Biology, Shanghai Institutes for Biological Sciences, Chinese Academy of Sciences, Shanghai 200031, China; ${ }^{2}$ Shanghai Key Laboratory of Molecular Andrology, Institute of Biochemistry and Cell Biology, Shanghai Institutes for Biological Sciences, Chinese Academy of Science, Shanghai 200031, China; ${ }^{3}$ Innovation Center for Cell Signaling Network, Institute of Biochemistry and Cell Biology, Shanghai Institutes for Biological Sciences, Chinese Academy of Science, Shanghai 200031, China; ${ }^{4}$ Shenzhen Center for Disease Control and Prevention, Shenzhen, Guangdong 518055, China; ${ }^{5}$ Department of Pathology, Fudan University Shanghai Cancer Center, Shanghai 200032, China; ${ }^{6}$ Department of Colorectal Surgery, Fudan University Shanghai Cancer Center, Shanghai 200032, China; ' Department of Radiation Oncology, First Affiliated Hospital of Wenzhou Medical College, Wenzhou, Zhejiang 325000, China
\end{abstract}

Pyruvate kinase M2 isoform (PKM2) catalyzes the last step of glycolysis and plays an important role in tumor cell proliferation. Recent studies have reported that PKM2 also regulates apoptosis. However, the mechanisms underlying such a role of PKM2 remain elusive. Here we show that PKM2 translocates to mitochondria under oxidative stress. In the mitochondria, PKM2 interacts with and phosphorylates Bcl2 at threonine (T) 69. This phosphorylation prevents the binding of Cul3-based E3 ligase to Bcl2 and subsequent degradation of Bcl2. A chaperone protein,

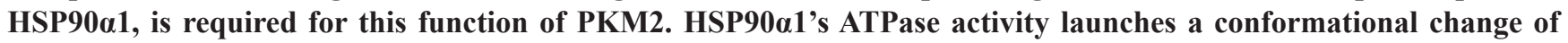
PKM2 and facilitates interaction between PKM2 and Bcl2. Replacement of wild-type Bcl2 with phosphorylation-deficient Bcl2 T69A mutant sensitizes glioma cells to oxidative stress-induced apoptosis and impairs brain tumor formation in an orthotopic xenograft model. Notably, a peptide that is composed of the amino acid residues from 389 to 405 of PKM2, through which PKM2 binds to Bcl2, disrupts PKM2-Bcl2 interaction, promotes Bcl2 degradation and impairs brain tumor growth. In addition, levels of Bcl2 T69 phosphorylation, conformation-altered PKM2 and Bcl2 protein correlate with one another in specimens of human glioblastoma patients. Moreover, levels of Bcl2 T69 phosphorylation and conformation-altered PKM2 correlate with both grades and prognosis of glioma malignancy. Our findings uncover a novel mechanism through which mitochondrial PKM2 phosphorylates Bcl2 and inhibits apoptosis directly, highlight the essential role of PKM2 in ROS adaptation of cancer cells, and implicate HSP90-PKM2-Bcl2 axis as a potential target for therapeutic intervention in glioblastoma.

Keywords: PKM2; Bcl2; HSP90; mitochondria; apoptosis; oxidative stress; tumorigenesis

Cell Research (2017) 27:329-351. doi:10.1038/cr.2016.159; published online 30 December 2017

\section{Introduction}

Cancer cells exhibit increased level of reactive oxygen species (ROS) than normal cells partly due to oncogenic

*These two authors contributed equally to this work.

Correspondence: Weiwei Yang

E-mail: weiweiyang@sibcb.ac.cn

Received 24 October 2016; revised 2 November 2016; accepted 10 November 2016; published online 30 December 2017 stimulation, increased metabolic activity and mitochondrial malfunction [1-3]. Under persistent oxidative stress, cancer cells become well-adapted to such stress through a set of mechanisms, which not only activate ROS-scavenging systems but also inhibit apoptosis [4]. A number of studies have suggested that such adaptation contributes to malignant transformation, metastasis and resistance to anticancer drugs [5-7]. Therefore, understanding mechanisms of ROS adaptation is important to efficiently kill cancer cells and overcome drug resistance. 
Pyruvate kinase (PK) catalyzes the final step of glycolysis and is comprised of four isoenzymes encoded by two distinct genes in mammals, PKLR and PKM. Alternative splicing of $P K M$ pre-mRNA results in the generation of pyruvate kinase M1 (PKM1) and M2 (PKM2) by the inclusion of exon 9 and exon 10 , respectively $[8$, 9]. PKM2, but not PKM1, is upregulated in most human cancers. Replacement of PKM2 with PKM1 in lung cancer cells inhibits aerobic glycolysis and tumor growth in nude mouse xenograft [10]. Activation of epidermal growth factor receptor (EGFR) in human glioma cells leads to increased glucose uptake and lactate production in a PKM2 expression-dependent manner [11]. Mediated by extracellular signal-regulated kinase (ERK)-dependent phosphorylation, PKM2 is capable of translocating to the nucleus upon EGRF activation [12]. In the nucleus, PKM2 binds to c-Src-phosphorylated $\beta$-catenin and enhances $\beta$-catenin's transactivation activity, promoting the expression of downstream oncogene cyclin D1 and the progression of cell cycle [13]. Under hypoxic conditions, prolyl-hydroxylated PKM2 interacts with HIF1a to induce glycolytic gene expression, which in turn enhances glucose metabolism in cancer cells [14]. These findings demonstrate the crucial roles of PKM2 in tumor cell proliferation.

Besides its important roles in promoting cell proliferation, PKM2 is also involved in the regulation of apoptosis. It has been shown that depletion of PKM2 expression by small interfering RNAs specifically against PKM2 results in decreased viability and increased apoptosis in multiple cancer cell lines [15]. Silencing of PKM2 in rat and human glioma spheroids enhances both apoptosis and differentiation [16]. In non-small cell lung cancer (NSCLC), PKM2 deficiency enhances ionizing radiation-induced apoptosis and autophagy in vitro and in vivo [17]. In addition to its anti-apoptotic function, PKM2 has been reported to translocate to the nucleus and induce a caspase-independent cell death in response to TT-232 [18]. These results indicate that PKM2 also plays instrumental roles in cell survival. However, the mechanism underlying such roles of PKM2 remains elusive.

B-cell lymphoma $2(\mathrm{Bcl} 2)$ is an anti-apoptotic member of the Bcl-2 family and is amplified in many human cancers [19]. The role of Bcl2 in ROS adaptation has been investigated in various laboratories. Ling et al. [20] demonstrated that ROS generation plays a vital role in the initiation of apoptotic cascade by proteasome inhibitor bortezomib. Overexpression of $\mathrm{Bcl} 2$ in prostate carcinoma cells diminishes bortezomib-induced apoptosis. Hildeman et al. [21] found that superoxide dismutase (SOD) mimetic MnTBAP decreases intracellular ROS and prevents apoptosis of activated T cells in vitro. Their study showed that MnTBAP increases the expression of
$\mathrm{Bcl}$, which is normally decreased by T-cell activation [21]. These findings indicate that Bcl2 plays an important role in ROS adaptation in different cell types. However, the mechanism through which $\mathrm{Bcl} 2$ is regulated during such adaptation is not well understood.

In this study, we report that PKM2 translocates to the mitochondria under oxidative stress. Mitochondrial PKM2 interacts with and phosphorylates Bcl2, which then prevents Cul3-RBX1 ligase-mediated degradation of Bcl2, enhances apoptosis resistance of tumor cells and promotes gliomagenesis.

\section{Results}

PKM2 translocates to the mitochondria and inhibits hydrogen peroxide-induced Bcl2 degradation and apoptosis

To investigate PKM2's role in ROS adaptation, we depleted endogenous PKM2 in U87 or U251 human glioblastoma multiforme (GBM) cells through the infection of a lentivirus expressing a specific shRNA against PKM2 (Supplementary information, Figure S1A). After hydrogen peroxide $\left(\mathrm{H}_{2} \mathrm{O}_{2}\right)$ or diamide (a thiol-oxidizing compound) treatment, U87 or U251 cells with PKM2 depletion (U87/shPKM2 or U251/shPKM2) had much more apoptotic cells than those cells expressing non-targeting shRNA (U87/shNT or U251/shNT), as determined by flow cytometry analysis of Annexin V-positive cells (Figure 1A and Supplementary information, Figure S1B). Similarly, caspase 3 activity was much more robust in U87/shPKM2 or U251/shPKM2 cells than that in U87/ shNT or U251/shNT cells after $\mathrm{H}_{2} \mathrm{O}_{2}$ treatment (Figure 1B). Cytochrome $c$ is released from the mitochondria to the cytosol, where it binds to Apafl to activate caspase cascades, during the early stage of mitochondria-dependent apoptosis [22]. Figure 1C showed that more cytochrome $c$ was detected in cytosolic fraction in U87/ shPKM2 or U251/shPKM2 cells than that in the cells expressing shNT after $\mathrm{H}_{2} \mathrm{O}_{2}$ treatment. Immune cells, such as Jurkat T cells, also express high levels of PKM2. To determine whether PKM2 has similar anti-apoptotic function in Jurkat $\mathrm{T}$ cells, we depleted endogenous PKM2 in Jurkat T cells and examined the effect of PKM2 depletion on $\mathrm{H}_{2} \mathrm{O}_{2}$-induced apoptosis. PKM2 depletion did not significantly increase the percentage of apoptotic cells in Jurkat T cells after $\mathrm{H}_{2} \mathrm{O}_{2}$ treatment (Supplementary information, Figure S1C), suggesting the cell-type specificity of PKM2 anti-apoptotic function. Taken together, these results demonstrate that PKM2 is essential for the resistance of glioma cells to oxidative stress-induced and mitochondria-dependent apoptosis.

To understand the mechanism of PKM2 regulation of such apoptosis, we examined subcellular localization of 
A
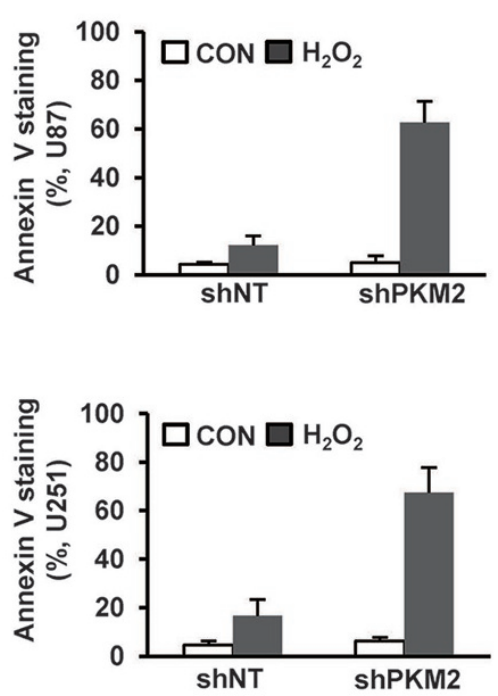

B
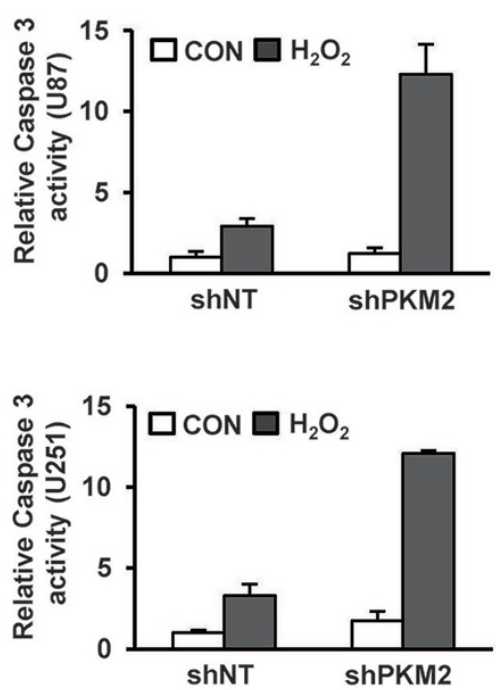

C

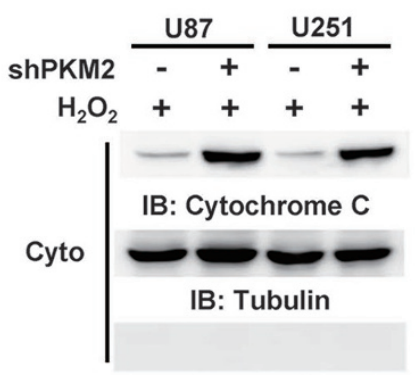

IB: VDAC

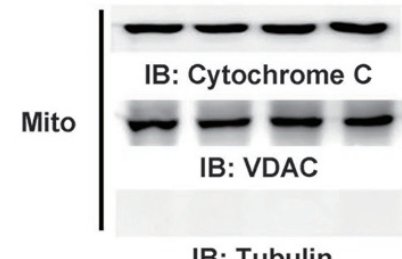

IB: Tubulin

E

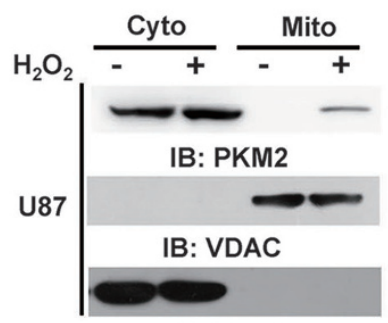

IB: Tubulin

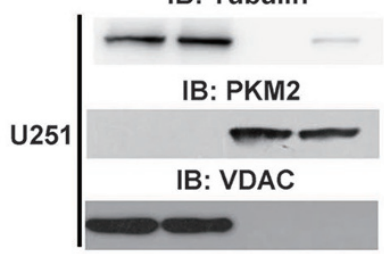

IB: Tubulin

G

F

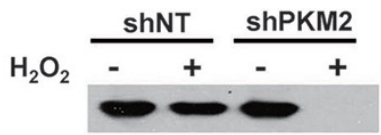

IB: Bcl2

IB: Bcl-xI

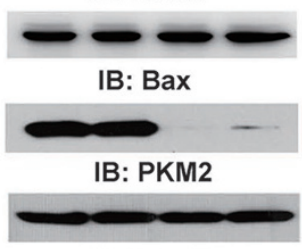

IB: Tubulin

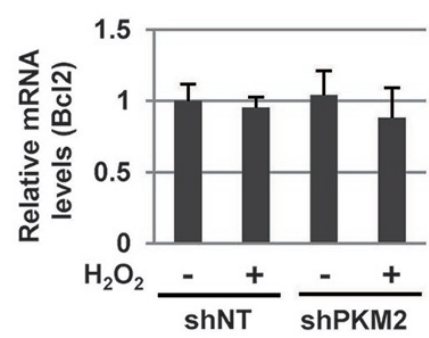

H

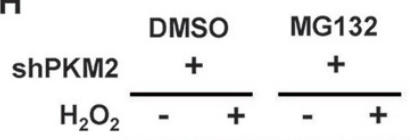

IB: Bcl2

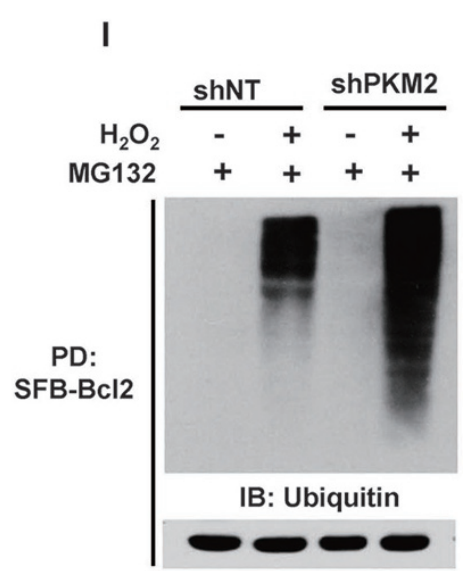

IB: Flag 
PKM2 after $\mathrm{H}_{2} \mathrm{O}_{2}$ treatment. Immunofluorescent analysis with anti-PKM2 or anti-VDAC (ubiquitously expressed and located in the outer mitochondrial membrane) antibody showed that $\sim 15 \%$ of PKM2 was translocated to the mitochondria in U87 cells upon $\mathrm{H}_{2} \mathrm{O}_{2}$ treatment (Figure 1D), which was supported by a subcellular fractionation analysis, showing that more PKM2 was detected in mitochondrial fraction of U87 or U251 cells after $\mathrm{H}_{2} \mathrm{O}_{2}$ treatment (Figure 1E). More thorough experiments of submitochondrial fractionation were also performed, showing that PKM2 was translocated to outer membrane of mitochondria upon $\mathrm{H}_{2} \mathrm{O}_{2}$ treatment (Supplementary information, Figure S1D). Thus, we speculated that PKM2 might regulate apoptosis via modulation of mitochondrial proteins. To test this possibility, we performed a mass spectrometry analysis of proteins associated with mitochondrial PKM2. Among them, three Bcl2 family proteins, including $\mathrm{Bcl} 2$, Bcl-xl and Bax, were identified (Supplementary information, Table S1). These proteins have been reported to regulate apoptosis by controlling mitochondrial membrane permeability [19]. Interactions between PKM2 and these proteins were further validated by co-immunoprecipitation analyses. Both $\mathrm{Bcl} 2$ and Bax could interact with PKM2, whereas Bcl-xl failed to do so (Supplementary information, Figure S1E). However, only Bcl2 protein level was markedly decreased in $\mathrm{H}_{2} \mathrm{O}_{2}$-treated U87/shPKM2 cells, whereas levels of Bcl$\mathrm{xl}$ or Bax proteins were not (Figure $1 \mathrm{~F}$ ). mRNA levels of $\mathrm{Bcl} 2$ showed no significant difference between U87/ shNT and U87/shPKM2 cells with or without $\mathrm{H}_{2} \mathrm{O}_{2}$ treatment, suggesting that PKM2 post-transcriptionally regulated Bcl2 (Figure 1G). Furthermore, treatment of a potent proteasome inhibitor MG132 abrogated $\mathrm{H}_{2} \mathrm{O}_{2}$-induced decrease of $\mathrm{Bcl} 2$ protein level in U87/shPKM2 cells (Figure 1H). These data confirmed that PKM2 depletion enhanced a proteasome-mediated degradation of $\mathrm{Bcl} 2$ induced by $\mathrm{H}_{2} \mathrm{O}_{2}$ treatment. Consistently, $\mathrm{H}_{2} \mathrm{O}_{2}$ induced more $\mathrm{Bcl} 2$ polyubiquitination in U87/shPKM2 cells than in U87/shNT cells (Figure 1I). These findings demonstrate that PKM2 translocates to the mitochondria and sustains $\mathrm{Bcl} 2$ protein stability, therefore enhancing the resistance of tumor cells to oxidative stress.

In addition to $\mathrm{H}_{2} \mathrm{O}_{2}$ treatment, we examined whether PKM2 depletion can enhance $\mathrm{Bcl} 2$ degradation under treatments of tumor necrosis factor $\alpha$ (TNF $\alpha$; inflammatory cytokine), paclitaxel (PTX; anti-cancer cytotoxic drug) or temozolomide (TMZ; an alkylating agent used for glioma treatment). As shown in Supplementary information, Figure S1F, PKM2 depletion enhanced TNF $\alpha$ and PTX-induced, but not TMZ-induced, Bcl2 degradation. More types of tumor cells, such as A549 lung cancer cells and HT29 colon cancer cells, were examined for PKM2's ability to stabilize Bcl2 protein. Consistent results were obtained, showing that PKM2 depletion promoted $\mathrm{H}_{2} \mathrm{O}_{2}$-induced degradation of $\mathrm{Bcl} 2$ in both $\mathrm{A} 549$ and HT29 cells (Supplementary information, Figure $\mathrm{S} 1 \mathrm{G})$.

To avoid the overexpression of target proteins, much less exogenous PKM2 or Bcl2 was expressed than those endogenous proteins in tumor cells through limiting infection efficiency (Supplementary information, Figure $\mathrm{S} 1 \mathrm{H})$.

\section{PKM2 interacts with and stabilizes Bcl2}

To investigate the mechanism through which PKM2 regulates $\mathrm{Bc12}$, a co-immunoprecipitation assay was performed to validate their interaction. Immunoblotting analyses of immunoprecipitated Bcl2 with an anti-PKM2 antibody (Figure 2A) or immunoprecipitated PKM2 with an anti-Bcl2 antibody (Supplementary information, Figure S2A) showed that endogenous PKM2 interacted with endogenous Bcl2 reciprocally, but not with succi-

Figure $1 \mathrm{PKM} 2$ translocates to the mitochondria and inhibits $\mathrm{H}_{2} \mathrm{O}_{2}$-induced $\mathrm{Bcl} 2$ degradation and apoptosis. Immunoprecipitation and immunoblotting analyses were performed with the indicated antibodies. Data are representative of at least three independent experiments. (A, B) U87 (top panel) or U251 (bottom panel) cells that stably express control shRNA (shNT) or shRNA against PKM2 (shPKM2) were treated with or without $\mathrm{H}_{2} \mathrm{O}_{2}(0.5 \mathrm{mM}, 24 \mathrm{~h})$. The cells were harvested for apoptosis analysis using Annexin V-Cy5 Apoptosis Kit (A) or caspase 3 activity analysis (B). Data represent the means \pm SD of three independent experiments. CON, control. (C) U87 or U251 cells with or without PKM2 depletion were treated with $\mathrm{H}_{2} \mathrm{O}_{2}(0.5$ $\mathrm{mM}, 24 \mathrm{~h}$ ) and harvested for cytosolic (Cyto) and mitochondrial (Mito) fractionation. (D) U87 cells were treated with or without $\mathrm{H}_{2} \mathrm{O}_{2}(0.5 \mathrm{mM}, 1 \mathrm{~h})$. Immunofluorescence analyses were performed using anti-PKM2 or anti-VDAC antibodies. Representative images were shown in left panel. Co-localization of two proteins in 300 cells was quantified using the software of Volocity (PerkinElmer, right panel). (E) U87 or U251 cells were treated with or without $\mathrm{H}_{2} \mathrm{O}_{2}(0.5 \mathrm{mM}, 1 \mathrm{~h})$. Cytosolic and mitochondrial fractions of the cells were prepared. (F) U87/shNT or U87/shPKM2 cells were treated with or without $\mathrm{H}_{2} \mathrm{O}_{2}(0.5 \mathrm{mM}, 4 \mathrm{~h})$. (G) U87/shNT or U87/shPKM2 cells were treated with or without $\mathrm{H}_{2} \mathrm{O}_{2}(0.5 \mathrm{mM}, 4 \mathrm{~h})$. Quantitative RT-PCR was performed to examine mRNA levels of $B c / 2$. Data represent the means \pm SD of three independent experiments. (H) U87/shPKM2 cells were pretreated with or without MG132 for $0.5 \mathrm{~h}$, followed by treatment of $\mathrm{H}_{2} \mathrm{O}_{2}(0.5 \mathrm{mM})$ for $4 \mathrm{~h}$. (I) U87/shNT or U87/shPKM2 cells were infected with a lentivirus expressing SFB-Bcl2. The cells were pretreated with MG132 for $0.5 \mathrm{~h}$ and then treated with or without $\mathrm{H}_{2} \mathrm{O}_{2}(0.5 \mathrm{mM}, 4 \mathrm{~h})$. Pull-down of SFB-Bcl2 was performed with streptavidin agarose beads. PD, pull-down. 
A

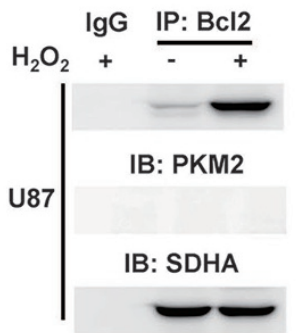

IB: $\mathrm{Bcl} 2$

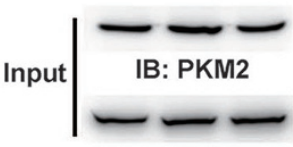

IB: SDHA

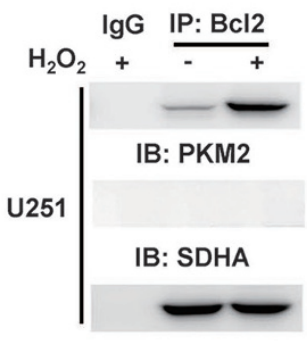

IB: Bcl2

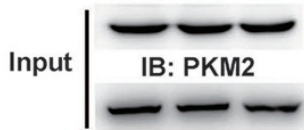

IB: SDHA
B

C

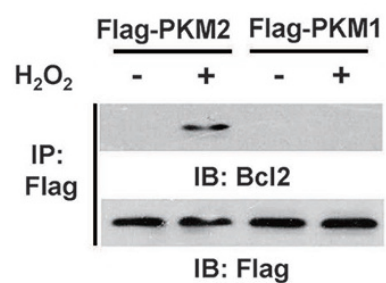

Input

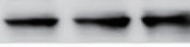

E

IB: Bcl2

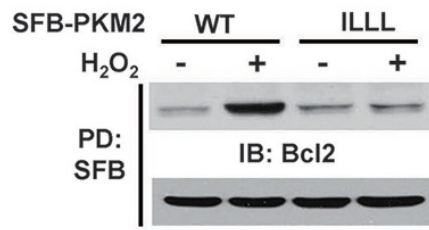

IB: Flag

Input

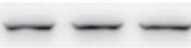

IB: Bcl2

H

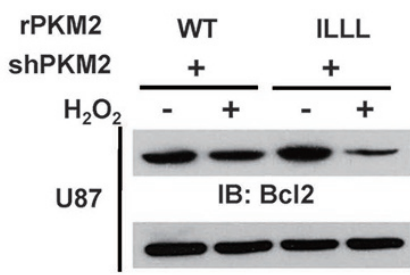

IB: Tubulin

I

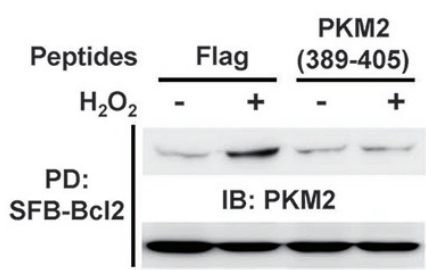

IB: Flag
F

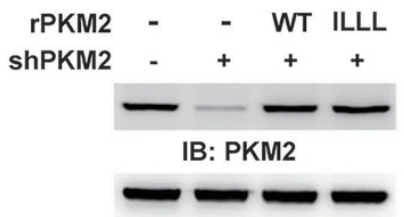

IB: Tubulin

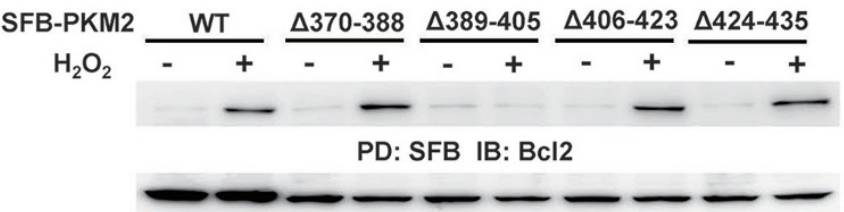

PD: SFB IB: Flag

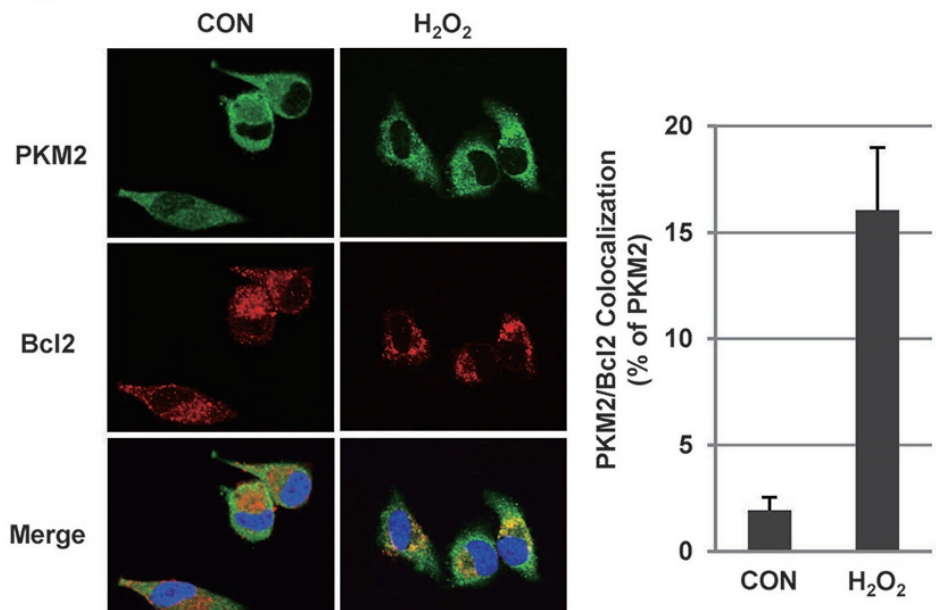

D

G

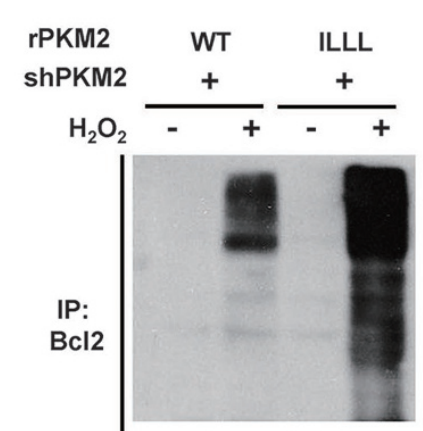

IB: Ubiquitin

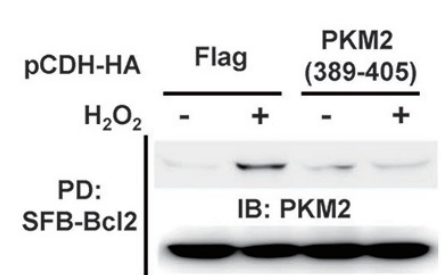

IB: Flag

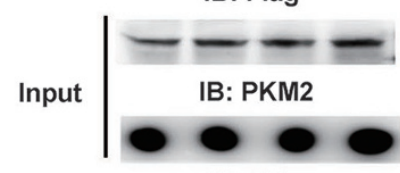

IB: HA
IB: Bcl2

K

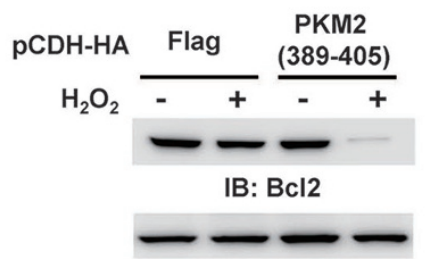

IB: Tubulin
IB: HA 
nate dehydrogenase (SDHA; used as a negative control) in both U87 and U251 cells upon $\mathrm{H}_{2} \mathrm{O}_{2}$ treatment. These observations were further supported by an immunofluorescence staining, showing that PKM2 colocalized with $\mathrm{Bcl} 2$ after $\mathrm{H}_{2} \mathrm{O}_{2}$ treatment (Figure 2B). We also examined the interaction between PKM2 and $\mathrm{Bcl} 2$ under various treatments or in various types of tumor cells. TNF $\alpha$ or PTX could induce the interaction between PKM2 and $\mathrm{Bcl} 2$, whereas TMZ failed to do so (Supplementary information, Figure S2B). Consistently, PKM2 interacted with Bc12 in A549 and HT29 cells, but not in Jurkat T cells, upon $\mathrm{H}_{2} \mathrm{O}_{2}$ treatment (Supplementary information, Figure S2C). We further explored the location of PKM2 interaction with $\mathrm{Bcl} 2$, as $\mathrm{PKM} 2$ and $\mathrm{Bcl} 2$ also locate in the nucleus besides the mitochondria. Subcellular fractionation experiments were performed, showing that PKM2 interacted with Bcl2 in the mitochondria, but not in the nucleus (Supplementary information, Figure S2D).

PKM1 shares high homology with PKM2 in protein sequence, as they differ from each other in only one exon. PKM2 contains exon 10, whereas PKM1 contains exon 9 [9]. To narrow searching range for Bcl2-interacting region on PKM2, we tested whether PKM1 interacts with Bcl2. U87 cells were infected with a lentivirus expressing Flag-tagged PKM2 (Flag-PKM2) or Flag-PKM1. Immunoblotting analyses of immunoprecipitated Flag-PKM2 or Flag-PKM1 with an anti-Bcl2 antibody showed that PKM1, unlike PKM2, did not interact with $\mathrm{Bcl} 2$ after $\mathrm{H}_{2} \mathrm{O}_{2}$ treatment (Figure 2C), suggesting that PKM2 may interact with $\mathrm{Bcl} 2$ through the region encoded by exon 10 of $P K M 2$. According to secondary structure of PKM2 exon 10, we constructed four S-Flag-Streptavidin-binding peptide (SFB)-tagged PKM2 mutants with the deletion of the indicated amino acid residues, including PKM2 $\triangle 370-388$, PKM2 $\Delta 389-$ 405, PKM2 $\Delta 406-423$ and PKM2 $\Delta 424-435$. Immunoblotting analyses of precipitated SFB-PKM2 proteins with anti-Bcl2 antibody showed that only PKM2 $\Delta 389$ 405 failed to bind to Bcl2 among PKM2 mutants (Figure 2D). Bcl2 has been reported to interact with other proteins through hydrophobic or ionic bonds [23]. According to amino acid properties, we constructed two PKM2 mutants, PKM2 ILLL, in which hydrophobic amino acids (isoleucine 389, leucine 392, leucine 394 and leucine 398) were mutated to alanine, and PKM2 EERR, in which ionic amino acids (glutamic acid 396, glutamic acid 397, arginine 399, and arginine 400) were mutated to alanine. Both PKM2 WT and PKM2 EERR interacted with Bcl2, whereas PKM2 ILLL failed to do so (Figure 2E and Supplementary information, Figure S2E).

To investigate whether PKM2-Bcl2 interaction is required for $\mathrm{Bcl} 2$ protein stability, we generated endogenous PKM2-depleted U87 cells with reconstituted expression of shRNA-resistant (r) PKM2 WT or rPKM2 ILLL (Figure 2F). Immunoblotting analyses of immunoprecipitated $\mathrm{Bcl} 2$ with anti-ubiquitin antibody showed that $\mathrm{H}_{2} \mathrm{O}_{2}$ treatment resulted in more polyubiquitination of Bcl2 in U87 cells expressing rPKM2 ILLL than in the cells expressing rPKM2 WT (Figure 2G). Consistently, $\mathrm{Bcl} 2$ protein levels were decreased in the cells expressing rPKM2 ILLL, but not in the cells expressing rPKM2 WT, after $\mathrm{H}_{2} \mathrm{O}_{2}$ treatment (Figure $2 \mathrm{H}$ ).

To test the specificity of PKM2 ILLL mutation, we examined pyruvate kinase and protein kinase activities of PKM2 ILLL mutant. As shown in Supplementary information, Figure S2F, PKM2 ILLL has comparable pyruvate kinase activity to PKM2 WT. We further examined the glycolytic activities of the corresponding PKM2

Figure 2 PKM2 interacts with and stabilizes Bcl2. Immunoprecipitation and immunoblotting analyses were performed with the indicated antibodies. Data are representative of at least three independent experiments. (A) U87 or U251 cells were treated with or without $\mathrm{H}_{2} \mathrm{O}_{2}(0.5 \mathrm{mM}, 1 \mathrm{~h})$. Succinate dehydrogenase (SDHA) that has not been reported to interact with PKM2 or $\mathrm{Bcl} 2$ was used as a negative control. (B) U87 cells were treated with or without $\mathrm{H}_{2} \mathrm{O}_{2}(0.5 \mathrm{mM}, 1 \mathrm{~h})$. Immunofluorescence was performed using anti-PKM2 and anti-Bcl2 antibodies. Representative images were presented in left panel. Co-localization of two proteins in 300 cells was quantified using the software of Volocity (right panel). (C) U87 cells were infected with a lentivirus expressing Flag-PKM1 or Flag-PKM2 and then treated with or without $\mathrm{H}_{2} \mathrm{O}_{2}(0.5 \mathrm{mM}, 1 \mathrm{~h})$. (D) U87 cells were infected with a lentivirus expressing SFB-PKM2 WT or the indicated deletion mutants and then treated with or without $\mathrm{H}_{2} \mathrm{O}_{2}(0.5$ mM, $1 \mathrm{~h})$. Pull-down of SFB-PKM2 proteins was performed with streptavidin agarose beads. (E) U87 cells stably expressing SFBPKM2 WT or ILLL were treated with or without $\mathrm{H}_{2} \mathrm{O}_{2}(0.5 \mathrm{mM}, 1 \mathrm{~h})$. Pull-down of SFB-PKM2 proteins was performed with streptavidin agarose beads. (F-H) U87 cells were depleted of endogenous PKM2 and then reconstituted with the expression of rPKM2 WT or rPKM2 ILLL (F). The cells were treated with or without $\mathrm{H}_{2} \mathrm{O}_{2}(0.5 \mathrm{mM})$ for $4 \mathrm{~h}(\mathbf{G}, \mathbf{H})$. The cells were pretreated with MG132 for $0.5 \mathrm{~h}$ before $\mathrm{H}_{2} \mathrm{O}_{2}$ treatment (G). (I) U87 cells that stably express SFB-Bcl2 were treated with or without $\mathrm{H}_{2} \mathrm{O}_{2}(0.5 \mathrm{mM}, 1 \mathrm{~h})$. SFB-Bcl2 complex was precipitated using streptavidin agarose beads. A peptide competition assay was performed by mixing precipitated SFB-Bcl2 complex with $1.0 \mu \mathrm{g}$ synthesized Flag or PKM2 $389-405$ peptides. (J, K) U87 cells that stably express SFB-Bcl2 were infected with a lentivirus expressing HA-tagged PKM2 389-405 peptide or Flag peptide and then treated with or without $\mathrm{H}_{2} \mathrm{O}_{2}(0.5 \mathrm{mM})$ for $1(\mathrm{~J})$ or $4 \mathrm{~h}(\mathrm{~K})$. SFB-Bcl2 complex was precipitated using streptavidin agarose beads $(\mathrm{J})$. Expression of Flag or PKM2 peptides was examined using dot blot assay with anti-HA antibody. 
ILLL-reconstituted cells. The data showed that similar glucose consumption and lactate production were detected in both PKM2 WT- and ILLL-reconstituted U87 cells (Supplementary information, Figure S2G). We previously reported that PKM2 could phosphorylate Histone H3 at threonine (T) 11 [24]. Thus, we examined the phosphorylation of Histone H3 T11 by PKM2 WT or ILLL. The data showed that both PKM2 WT and ILLL could phosphorylate Histone H3 T11 at similar levels (Supplementary information, Figure S2H). Together, these data suggested that PKM2 ILLL only lost the binding to Bcl2, but still reserved its pyruvate kinase and protein kinase activities.

Peptide medicine is recognized for being highly selective and efficacious, and at the same time, relatively safe and well tolerated. We next investigated the possibility of the peptide that is composed of amino acids ranging from 389 to 405 of PKM2 as an anti-tumor drug. U87 cells were infected with a lentivirus expressing SFB-Bcl2. Precipitated SFB-Bcl2 complex was incubated with synthesized Flag or PKM2 389-405 peptides. PKM2 389405 peptide remarkably disrupted $\mathrm{H}_{2} \mathrm{O}_{2}$-induced interaction between PKM2 and Bcl2, whereas Flag peptides could not (Figure 2I).

To confirm actual binding of PKM2 peptides to Bcl2, biotinylated PKM2 or Flag peptides were synthesized and incubated with U87 lysates or bacteria-purified recombinant Bcl2. As shown in Supplementary information, Figure S2I and S2J, endogenous Bcl2 or recombinant $\mathrm{Bcl} 2$ could be co-precipitated with biotinylated PKM2 peptides, but not with biotinylated Flag peptides, in a dose-dependent manner. In addition, the more PKM2 was expressed, the less Bcl2 was pulled down by biotinylated PKM2 peptides (Supplementary information, Figure S2K).

Inhibition of PKM2-Bcl2 interaction by PKM2 peptides was further examined in cells. U87 cells that stably express SFB-Bc12 were infected with a lentivirus expressing PKM2 389-405 or Flag peptides. Expression of PKM2 peptides significantly inhibited $\mathrm{H}_{2} \mathrm{O}_{2}$-induced interaction between PKM2 and $\mathrm{Bcl} 2$, and caused subsequent degradation of Bcl2 (Figure 2J and 2K). Expression levels of PKM2 or Flag peptides were examined using dot blot assay, showing that PKM2 and Flag peptides had similar expression levels (Figure 2J and 2K). These results implicate a therapeutic potential of PKM2 389405 peptide as an anti-cancer drug.

HSP90a1 alters PKM2 conformation and facilitates interaction between PKM2 and Bcl2

To investigate mechanism through which PKM2 interacts with $\mathrm{Bcl} 2$, we tested whether PKM2 interacts with
Bc12 directly. Recombinant His-Bcl2 and GST-PKM2 were purified from bacteria. Purities of two proteins were examined by gel staining with Coomassie Blue (Supplementary information, Figure S3A). GST pulldown assays were performed by mixing PKM2 and Bcl2 or PKM2 and commercial Histone H3 (NEB) that has been reported to interact with PKM2 in vitro [24]. The data showed that $\mathrm{Bcl} 2$ could not bind to PKM2 in vitro, whereas Histone H3 could do so (Figure 3A). Analysis of PKM2 structure implied that PKM2 389-405 fragment, especially four hydrophobic amino acids (I389, L392, L394 and L398) of PKM2 that are essential for PKM2$\mathrm{Bcl} 2$ interaction, are mostly buried inside (Supplementary information, Figure S3B). Thus, we proposed that PKM2 structure should be altered to expose those critical amino acids. To search for potential isomerase, we performed a mass spectrum analysis of proteins associated with total PKM2 after $\mathrm{H}_{2} \mathrm{O}_{2}$ treatment. HSP90 $\alpha 1$ was identified to be the only associated protein that can alter protein conformation (Supplementary information, Table S2). Interaction between HSP90 $\alpha 1$ and PKM2 was further validated by a co-immunoprecipitation assay, showing that $\mathrm{HSP} 90 \alpha 1$ interacted with PKM2 after $\mathrm{H}_{2} \mathrm{O}_{2}$ treatment (Figure 3B).

HSP90 $\alpha 1$, an isoform of HSP90, is a chaperone protein that assists other proteins to fold properly, stabilizes proteins against heat stress and aids in protein degradation. To determine whether HSP90 $\alpha 1$ is required for PKM2-Bcl2 interaction, we depleted HSP90 $\alpha 1$ in U87 cells with a shRNA against HSP90AA1 (Supplementary information, Figure S3C). Immunoblotting analyses of immunoprecipitated Bcl2 with anti-PKM2 antibody showed that HSP90 $\alpha 1$ depletion abrogated the interaction between PKM2 and Bcl2 (Figure 3C). To map the interacting region of $\mathrm{HSP} 90 \alpha 1$ and PKM2, three deletion mutants of HSP90 1 1 were constructed (Figure 3D), including $\mathrm{N}$-terminal deletion mutant $(\Delta \mathrm{N})$, middle domain mutant (MD) and $\mathrm{C}$-terminal deletion mutant $(\Delta \mathrm{C})$. Among three HSP90a1 mutants, only HSP90 $\alpha 1$ MD could bind to PKM2 (Figure 3E). To further pinpoint the exact interacting region on HSP90 $\alpha 1$, three more deletion mutants of HSP90 $\alpha 1$ were constructed according to crystal structure of HSP90 $\alpha 1$ [25], including HSP90 $\alpha 1$ 1-293, 1-360 and 1-469. Co-immunoprecipitation analyses of HSP90 $\alpha 1$ WT and mutants showed that only HSP90 $\alpha 1$ 1-293 lost the binding to PKM2 among the mutants under $\mathrm{H}_{2} \mathrm{O}_{2}$ treatment (Figure $3 \mathrm{~F}$ ). The region spanning amino acids $327-340$ of HSP90 $\beta$, which is homologous to the region spanning amino acids $347-360$ of HSP90 $\alpha 1$, has been reported to be involved in the binding to its client protein AKT [26]. Thus, we tested whether amino acids 347-360 of HSP90 1 are required for its 
A

$\begin{array}{rrrrr}\text { His-Bcl2 } & - & - & + & + \\ \text { Histone H3 } & + & + & - & - \\ \text { GST-PKM2 } & - & + & - & +\end{array}$

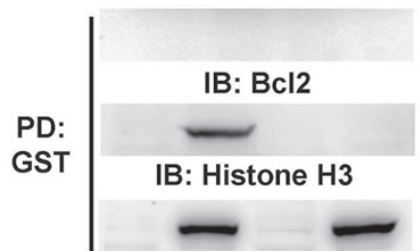

IB: PKM2

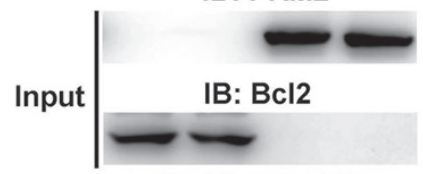

IB: Histone H3

D

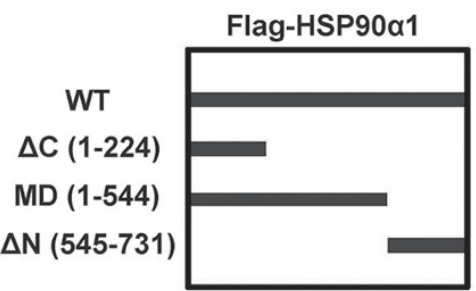

G

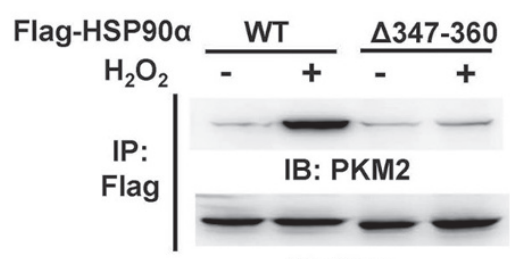

IB: Flag

Input

IB: PKM2

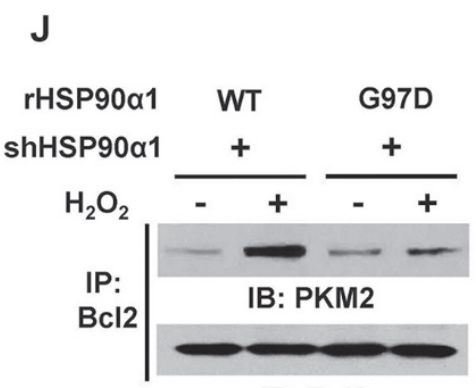

IB: Bcl2

B

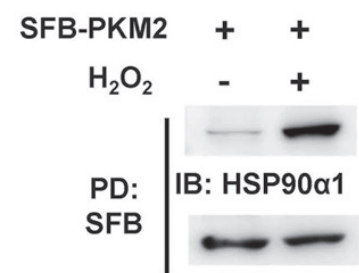

IB: Flag

Input

IB: HSP90a1

E

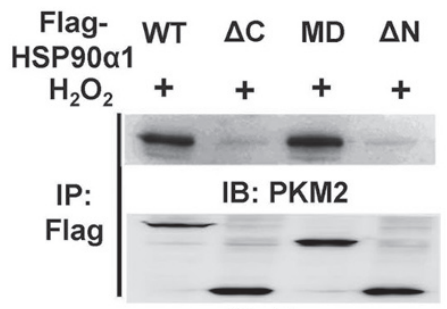

IB: Flag

Input

IB: PKM2

H

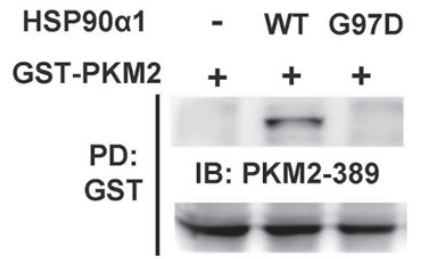

IB: GST

Input

IB: HSP90a1

K

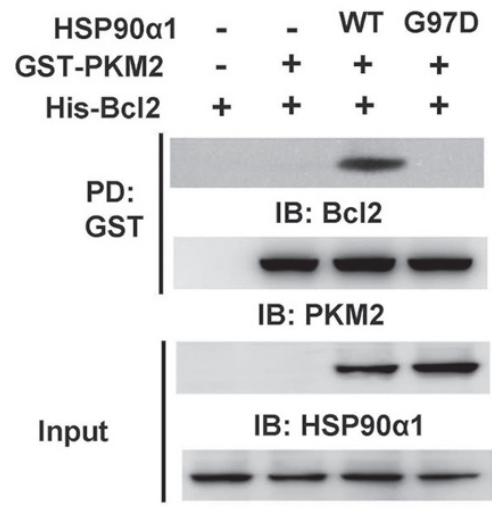

IB: Bcl2
C

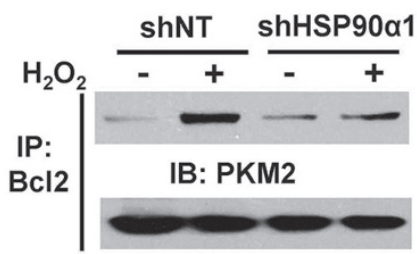

IB: Bcl2

Input

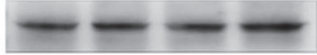

IB: PKM2

F
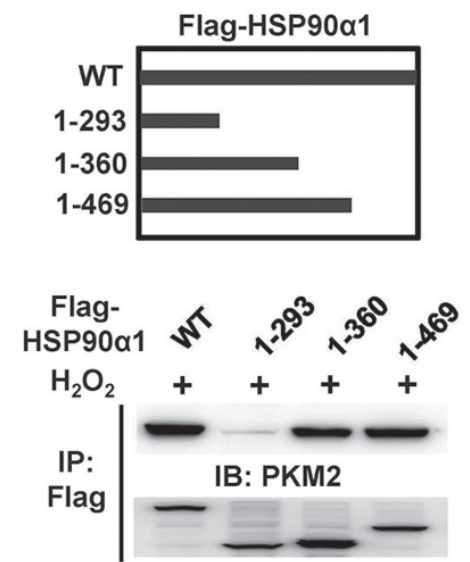

IB: Flag

Input

IB: PKM2

I

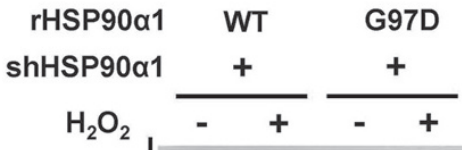

PD:

SFB-PKM2

IB: PKM2-389

IB: Flag

L

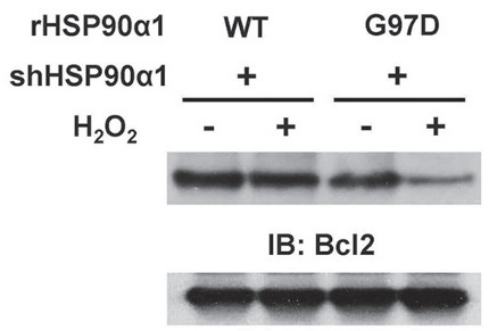

IB: Tubulin 
binding to PKM2. HSP90 1 deletion mutant, HSP901 $\Delta 347-360$ lost the binding to PKM2 after $\mathrm{H}_{2} \mathrm{O}_{2}$ treatment (Figure 3G). Meanwhile, we also mapped the region of HSP90 1-PKM2 interaction on PKM2. Supplementary information, Figure S3D showed that both PKM2 $\Delta 389$ 405 and PKM2 $\Delta 406-423$ lost their binding to HSP90 $\alpha 1$. Ionic bonds are often involved in HSP90 substrate binding [26]. Considering simultaneous surface exposure of the interacting amino acids of PKM2, four ionic amino acids (R399, R400, D407 and E410) of PKM2, which are located on the surface of PKM2 protein, were mutated to alanine (PKM2 RRDE). Co-immunoprecipitation analyses showed that PKM2 WT interacted with HSP90 $\alpha 1$ upon $\mathrm{H}_{2} \mathrm{O}_{2}$ treatment, whereas PKM2 RRDE mutant failed to do so (Supplementary information, Figure $\mathrm{S} 3 \mathrm{E})$. Together, these results demonstrated that amino acids 347-360 of HSP90 $\alpha 1$ are involved in the binding to PKM2, and that PKM2 is likely a client protein of HSP90 1 .

HSP90 1 's ATPase activity often induces conformational change in client proteins, thereby causing their activation [27]. To determine whether HSP90 $\alpha 1$ alters PKM2 conformation, we performed chaperon assay by mixing recombinant GST-PKM2 with HSP90 1 WT or HSP90 $\alpha 1$ G97D (ATPase activity dead mutant), in which glycine 97 was mutated to aspartic acid. Immunoblotting analyses of precipitated GST-PKM2 with a PKM2389 antibody, which specifically recognizes PKM2 389405 fragment that is buried inside the protein before $\mathrm{H}_{2} \mathrm{O}_{2}$ treatment, showed that GST-PKM2 could only be recognized by PKM2-389 antibody in the presence of HSP90 1 WT, but not HSP90 1 1 G97D (Figure 3H), suggesting that PKM2 conformation was altered by
HSP90 $\alpha 1$ to expose PKM2 389-405 fragment. We further examined this result in cells. U87 cells were depleted of endogenous HSP90 $\alpha 1$ and reconstituted with the expression of rHSP90 $\alpha 1$ WT or rHSP90 $\alpha 1$ G97D (Supplementary information, Figure S3F). Inhibition of HSP90 activity by HSP90 $\alpha 1$ G97D expression was confirmed by examining the expression of HSP90 $\alpha 1$ substrate proteins, AKT1 and Chk1. rHSP90 $\alpha 1$ G97D expression significantly inhibited the expression of AKT1 and Chk1 (Supplementary information, Figure S3G). Immunoblotting analyses of the precipitated SFB-PKM2 with PKM2389 antibody showed that $\mathrm{H}_{2} \mathrm{O}_{2}$-induced alteration of PKM2 conformation could only be detected in the cells expressing rHSP90 $\alpha 1 \mathrm{WT}$, but not in the cells expressing rHSP90a1 G97D (Figure 3I).

To determine whether HSP90 1 1-mediated conformational change of PKM2 is required for PKM2-Bcl2 interaction, co-immunoprecipitation assay was performed using Bcl2 antibody in HSP90 1 1-depleted U87 cells that were reconstituted with the expression of rHSP90 $\alpha 1 \mathrm{WT}$ or G97D. As shown in Figure 3J, PKM2 interacted with Bcl2 in cells expressing rHSP90 $1 \mathrm{WT}$, but not in cells expressing rHSP90 $\alpha 1 \mathrm{G} 97 \mathrm{D}$, after $\mathrm{H}_{2} \mathrm{O}_{2}$ treatment. This result was further supported by an HSP90 $\alpha 1$-mediated chaperon assay followed by a GST pull-down assay, showing that PKM2 could only interact with Bcl2 in the presence of HSP90 $\alpha 1$ WT, but not HSP90 $\alpha 1$ G97D (Figure $3 \mathrm{~K}$ ). Consistently, $\mathrm{H}_{2} \mathrm{O}_{2}$ treatment induced more degradation of $\mathrm{Bcl} 2$ in the cells expressing rHSP90 $\alpha 1$ G97D than in the cells expressing rHSP90 $\alpha 1$ WT (Figure 3L).

HSP90 1 1-mediated conformational change of PKM2 was also confirmed using an HSP90 inhibitor (17-AAG), which could efficiently inhibit expression of AKT1

Figure 3 HSP90 1 alters PKM2 conformation and facilitates the interaction between PKM2 and Bcl2. Immunoprecipitation and immunoblotting analyses were performed with the indicated antibodies. Data are representative of at least three independent experiments. (A) GST pull-down assay was performed by mixing bacteria-purified recombinant GST-PKM2 and His-Bcl2, or GST-PKM2 and Histone $\mathrm{H} 3$ at $4{ }^{\circ} \mathrm{C}$ for $4 \mathrm{~h}$. GST-PKM2 was precipitated using glutathione agarose beads. (B) U87 cells that stably express SFB-PKM2 were treated with or without $\mathrm{H}_{2} \mathrm{O}_{2}(0.5 \mathrm{mM}, 1 \mathrm{~h})$. SFB-PKM2 was pulled down using streptavidin agarose beads. (C) U87 cells that stably express shNT or shHSP90 1 were treated with or without $\mathrm{H}_{2} \mathrm{O}_{2}$ (0.5 mM, $1 \mathrm{~h})$. (D) Flag-HSP90 $1 \mathrm{WT}$ and Flag-HSP90 1 deletion mutants, HSP90 $1 \Delta \mathrm{C}(1-224), \mathrm{MD}(1-544)$ and $\Delta \mathrm{N}(545-$ 731), used in the experiments are represented as black bars. (E) U87 cells were infected with a lentivirus expressing FlagHSP90 1 WT or Flag-HSP90 1 deletion mutants and then treated with $\mathrm{H}_{2} \mathrm{O}_{2}(0.5 \mathrm{mM}, 1 \mathrm{~h})$. (F) U87 cells were infected with

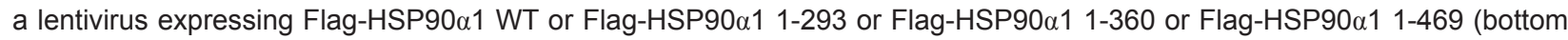
panel). Flag-HSP90 1 WT and Flag-HSP90 1 truncation mutants, HSP90 1 1-293, 1-360 and 1-469, used in the experiments are represented as black bars (top panel). (G) U87 cells were infected with a lentivirus expressing Flag-HSP90 1 WT or Flag-HSP90 $\alpha 1$ 4347-360. (H) Chaperone assay was performed by mixing GST-PKM2 and recombinant HSP90 1 WT or G97D. After the reaction, GST-PKM2 was pulled down using glutathione agarose beads. (I) U87 cells were depleted of endogenous HSP90 1 and then reconstituted with the expression of rHSP90 1 WT or G97D. The cells were further infected with a lentivirus expressing SFB-PKM2 and then treated with or without $\mathrm{H}_{2} \mathrm{O}_{2}(0.5 \mathrm{mM}, 1 \mathrm{~h})$. (J) HSP90a1-depleted U87 cells were reconstituted with the expression of rHSP90a1 WT or G97D and then treated with or without $\mathrm{H}_{2} \mathrm{O}_{2}(0.5 \mathrm{mM}, 1 \mathrm{~h})$. (K) Chaperone assay was performed by mixing recombinant HSP90 1 WT or G97D and GST-PKM2. After the reaction, GSTPKM2 were pulled down using glutathione agarose beads and incubated with His-Bcl2 at $4{ }^{\circ} \mathrm{C}$ for $4 \mathrm{~h}$. (L) HSP90 1 -depleted U87 cells that were reconstituted with the expression of rHSP90 1 WT or G97D were treated with or without $\mathrm{H}_{2} \mathrm{O}_{2}(0.5 \mathrm{mM}, 4 \mathrm{~h})$. 
and Chk1 (Supplementary information, Figure S3H). As shown in Supplementary information, Figure S3I and S3J, pretreatment of 17-AAG markedly inhibited $\mathrm{H}_{2} \mathrm{O}_{2}$-induced alteration of PKM2 conformation and PKM2-Bcl2 interaction. Consistently, $\mathrm{H}_{2} \mathrm{O}_{2}$-induced degradation of $\mathrm{Bcl} 2$ was markedly enhanced by $17-\mathrm{AAG}$ treatment (Supplementary information, Figure S3K).

\section{PKM2 phosphorylates Bcl2 at T69}

It has been reported that $\mathrm{Bcl} 2$ stability could be regulated by phosphorylation [28]. To investigate whether $\mathrm{PKM} 2$ regulates $\mathrm{Bcl} 2$ phosphorylation, we performed mass spectrometry analysis of $\mathrm{Bcl} 2$ phosphorylation in U87 cells after $\mathrm{H}_{2} \mathrm{O}_{2}$ treatment, showing that threonine (T) 69 and serine (S) 70 of Bcl2 were phosphorylated after $\mathrm{H}_{2} \mathrm{O}_{2}$ treatment. Immunoblotting analyses of the precipitated SFB-Bcl2 with anti-Bcl2 pT69 and anti-Bcl2 pS70 antibodies showed that PKM2 depletion significantly blocked Bc12 T69 phosphorylation, but not Bcl2 S70 phosphorylation (Figure 4A and Supplementary information, Figure S4A). Regulation of Bc12 T69 phosphorylation by PKM2 was further verified in more types of tumor cells or under more treatments. Both TNF $\alpha$ - and PTX-induced Bc12 T69 phosphorylation were significantly inhibited by PKM2 depletion, whereas TMZ treatment could not even induce Bcl2 T69 phosphorylation (Supplementary information, Figure $\mathrm{S} 4 \mathrm{~B}$ ). $\mathrm{H}_{2} \mathrm{O}_{2}$-induced $\mathrm{Bcl} 2$ T69 phosphorylation was blocked by PKM2 depletion in A549 or HT29 cells, whereas Bcl2 T69 phosphorylation could not even be induced in Jurkat $\mathrm{T}$ cells after $\mathrm{H}_{2} \mathrm{O}_{2}$ treatment (Supplementary information, Figure S4C).

Previous studies from our and other groups have reported that PKM2 is capable of phosphorylating proteins as a protein kinase [24]. We next tested whether $\mathrm{PKM} 2$ phosphorylates Bcl2. In vitro kinase assays were performed by mixing ${ }^{32}$ p-labeled PEP with recombinant PKM2 or PKM1 and Bcl2 WT or phosphorylation-deficient $\mathrm{Bcl} 2$ mutants. Figure $4 \mathrm{~B}$ and $4 \mathrm{C}$ showed that PKM2, but not PKM1, could phosphorylate Bcl2, and that Bcl2 T69A was resistant to phosphorylation, whereas Bcl2 S70A could still be phosphorylated. Phosphorylation of Bcl2 $\mathrm{T} 69$ by PKM2 was further validated by LC-MS/MS analyses, showing that Bcl2 T69 was the only residue phosphorylated by PKM2 (Figure 4D and Supplementary information, Figure S4D). To further verify Bcl2 T69 phosphorylation by PKM2 in cells, U87 cells with depleted PKM2 and reconstituted expression of rPKM2 WT or ILLL were infected with a lentivirus expressing SFB-Bc12. Immunoblotting analyses of the precipitated SFB-Bcl2 with anti-Bc12 pT69 antibody showed that Bcl2 T69 was phosphorylated in cells expressing rPKM2 WT, but not in cells expressing rPKM2
ILLL, after $\mathrm{H}_{2} \mathrm{O}_{2}$ treatment (Figure 4E). Together, these data clearly indicated that PKM2 phosphorylated Bcl2 at T69 after $\mathrm{H}_{2} \mathrm{O}_{2}$ treatment.

We further tested whether PKM2-dependent Bcl2 phosphorylation is required to sustain $\mathrm{Bcl} 2$ protein level. U87 cells were infected with a lentivirus expressing SFB-Bcl2 WT or T69A. Immunoblotting analyses of the precipitated SFB-Bcl2 with an anti-ubiquitin antibody showed that $\mathrm{Bc} 2 \mathrm{~T} 69 \mathrm{~A}$ had more polyubiquitination than $\mathrm{Bcl} 2 \mathrm{WT}$ after $\mathrm{H}_{2} \mathrm{O}_{2}$ treatment (Figure 4F). Consistently, $\mathrm{H}_{2} \mathrm{O}_{2}$ treatment resulted in more degradation of $\mathrm{Bcl} 2 \mathrm{~T} 69 \mathrm{~A}$ than that of Bcl2 WT (Figure 4G).

PKM2-dependent Bcl2 T69 phosphorylation prevents the binding of Cul3-based E3 ligase to Bcl2

It has been reported that $\mathrm{Bcl} 2$ could be ubiquitinated by Cul3-based E3 ligase and subsequently degraded [29]. To investigate the mechanism through which PKM2 stabilizes Bc12 protein, we examined whether PKM2 interferes with recruitment of Cul3-based E3 ligase to Bcl2. U87 cells were depleted of PKM2 and reconstituted with expression of rPKM2 WT or ILLL. Immunoblotting analyses of immunoprecipitated $\mathrm{Bcl} 2$ with anti-Cul3 and anti-Rbx 1 antibodies showed that $\mathrm{Bcl} 2$ interacted with both $\mathrm{Cul} 3$ and $\mathrm{Rbx} 1$ in cells expressing rPKM2 ILLL, but not in cells expressing rPKM2 WT, after $\mathrm{H}_{2} \mathrm{O}_{2}$ treatment (Figure 5A), suggesting that PKM2 interacts with $\mathrm{Bcl} 2$ and prevents binding of $\mathrm{E} 3$ ligase to $\mathrm{Bcl} 2$. We next examined whether Bcl 2 T69 phosphorylation is required to prevent the recruitment of E3 ligase. Figure 5B showed that Bcl2 T69A was associated with more $\mathrm{Cul} 3$ and $\mathrm{Rbx} 1$ than $\mathrm{Bcl} 2$ WT after $\mathrm{H}_{2} \mathrm{O}_{2}$ treatment. In contrast, phosphorylation-mimic mutant of Bcl2, Bcl2 T69E, interacted with much less $\mathrm{Cul} 3$ and $\mathrm{Rbx} 1$ than $\mathrm{Bcl} 2 \mathrm{WT}$ in $\mathrm{H}_{2} \mathrm{O}_{2}$-treated $\mathrm{U} 87$ cells with PKM2 depletion (Figure $5 \mathrm{C})$.

To further demonstrate whether Cul3-based E3 ligase is involved in $\mathrm{H}_{2} \mathrm{O}_{2}$-induced $\mathrm{Bcl} 2$ degradation, we depleted Cul3 using siRNA against CUL3 (siCul3) in U87 cells with depleted PKM2 and reconstituted expression of rPKM2 ILLL, or in U87 cells expressing Bcl2 T69A. Immunoblotting analyses of immunoprecipitated $\mathrm{Bcl} 2$ or precipitated SFB-Bcl2 with anti-ubiquitin antibodies showed that $\mathrm{Cul} 3$ depletion remarkably inhibited $\mathrm{H}_{2} \mathrm{O}_{2}$-induced polyubiquitination of $\mathrm{Bcl} 2$ in cells expressing rPKM2 ILLL or $\mathrm{H}_{2} \mathrm{O}_{2}$-induced polyubiquitination of Bcl2 T69A (Figure 5D and 5E). Consistently, Cul3 depletion blocked $\mathrm{H}_{2} \mathrm{O}_{2}$-induced degradation of $\mathrm{Bcl} 2$ in cells expressing rPKM2 ILLL or $\mathrm{H}_{2} \mathrm{O}_{2}$-induced degradation of Bcl2 T69A (Figure 5F and 5G). Taken together, these results demonstrate that PKM2-dependent phosphorylation of Bcl2 T69 prevents the binding of Cul3-Rbx1 ligase to 
A

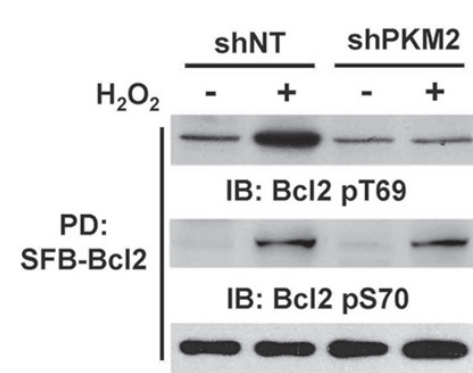

IB: Flag
B

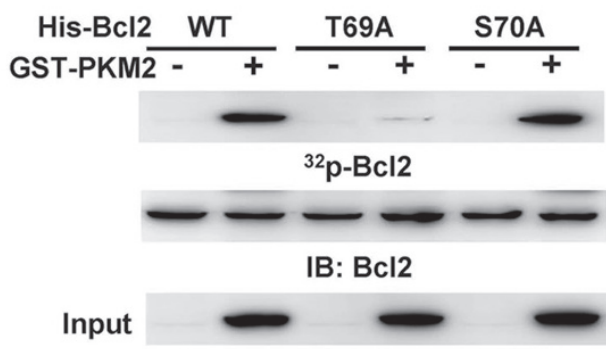

IB: PKM2
C

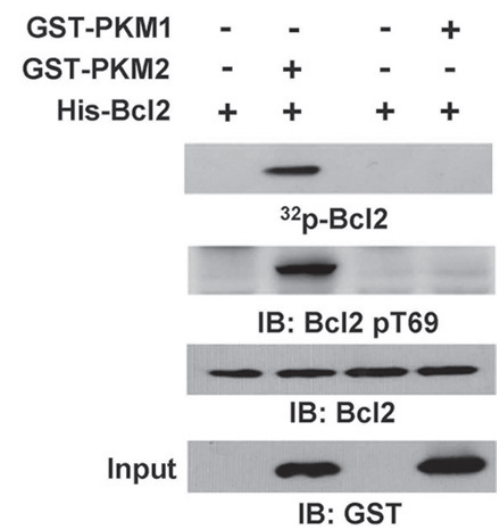

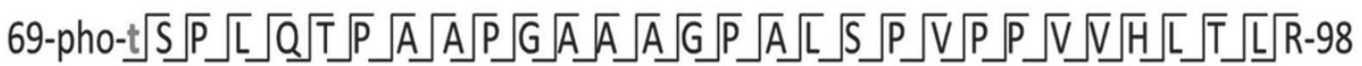

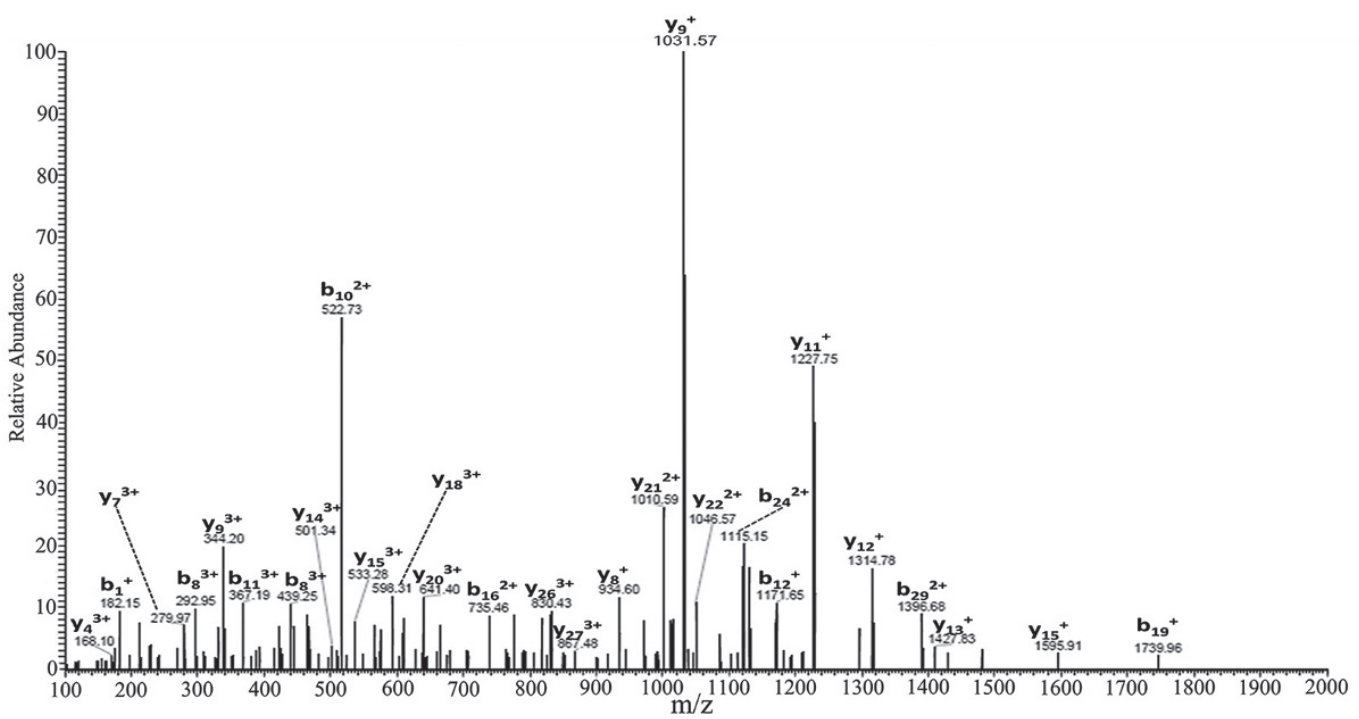

E

$\mathbf{F}$

G

$\underset{\mathrm{H}_{2} \mathrm{O}_{2}}{\mathrm{SFB}-\mathrm{Bcl} 2} \frac{\mathrm{WT}}{-++} \frac{\mathrm{T} 69 \mathrm{~A}}{-+}$

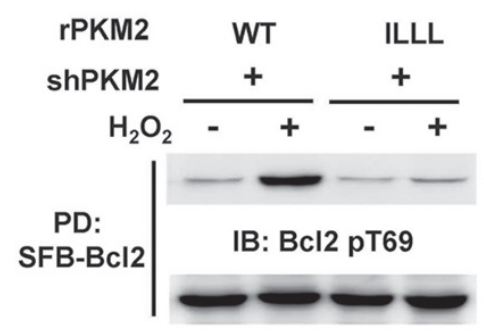

IB: Flag

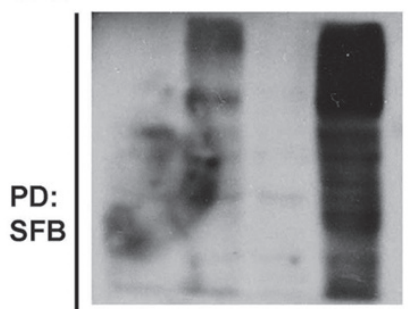

IB: Ubiquitin

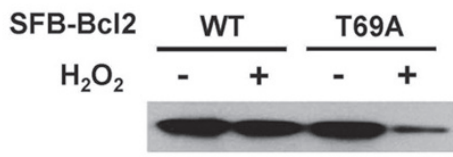

IB: Flag

IB: Tubulin

IB: Flag 
$\mathrm{Bcl} 2$, therefore inhibiting $\mathrm{Bcl} 2$ degradation.

Phosphorylation of Bcl2 T69 by PKM2 enhances resistance of tumor cells to oxidative stress and promotes gliomagenesis

To explore the biological function of PKM2-dependent Bcl2 T69 phosphorylation, U87 or U251 cells were depleted of PKM2 and reconstituted with the expression of rPKM2 WT or ILLL (Figure 2F and Supplementary information, Figure S5A); U87 or U251 cells were depleted of $\mathrm{Bcl} 2$ and reconstituted with the expression of rBcl2 WT or T69A (Supplementary information, Figure S5B and S5C). Apoptosis analysis using Annexin V-Cy5 staining showed that more apoptotic cells were detected in cells expressing rPKM2 ILLL or $\mathrm{rBcl} 2 \mathrm{~T} 69 \mathrm{~A}$ than in cells expressing rPKM2 WT or rBcl2 WT after $\mathrm{H}_{2} \mathrm{O}_{2}$ treatment (Figure 6A and 6B, Supplementary information, Figure S5D and S5E, top panel). Consistently, $\mathrm{H}_{2} \mathrm{O}_{2}$ treatment led to the stronger caspase 3 activity in cells expressing rPKM2 ILLL or rBcl2 T69A than that in cells expressing rPKM2 WT or $\mathrm{rBcl} 2 \mathrm{WT}$ after $\mathrm{H}_{2} \mathrm{O}_{2}$ treatment (Figure 6A and 6B, Supplementary information, Figure S5D and S5E, bottom panel). In addition, effects of phosphorylation-mimic Bcl2 T69E on apoptosis were examined. As shown in Supplementary information, Figure $\mathrm{S} 5 \mathrm{~F}$, expression of $\mathrm{rBcl} 2 \mathrm{~T} 69 \mathrm{E}$ markedly abrogated $\mathrm{H}_{2} \mathrm{O}_{2}$-induced apoptosis of PKM2-depleted U87 cells. Together, these data demonstrate that PKM2-dependent Bcl2 T69 phosphorylation is essential for oxidative stress adaptation of glioma cells.

To further explore the cellular functions of PKM2-dependent $\mathrm{Bcl} 2$ phosphorylation, apoptosis analyses were performed in U87 cells under various types of treatments. As shown in Supplementary information, Figure S5G and
$\mathrm{S} 5 \mathrm{H}$, more apoptotic cells were observed in PKM2-depleted U87 cells with reconstituted expression of rPKM2 ILLL or Bcl2-depleted U87 cells with reconstituted expression of $\mathrm{rBcl} 2 \mathrm{~T} 69 \mathrm{~A}$ than those in the cells expressing rPKM2 WT or rBcl2 WT after TNF $\alpha$ or PTX treatment. It has been extensively reported that the treatment of TNF $\alpha$ or PTX induces significant ROS production and ROS plays an essential role in TNF $\alpha$ - or PTX-induced apoptosis [30, 31]. Thus, PKM2-dependent Bcl2 regulation induced by TNF $\alpha$ or PTX is very likely mediated by increased ROS after the treatment. This hypothesis was simply tested by using the antioxidant $N$-acetyl-cysteine (NAC) to decrease intracellular ROS levels. The data showed that NAC treatment significantly abrogated TNF $\alpha$ - or PTX-induced apoptosis in PKM2-depleted U87 cells, and that TNF $\alpha$ - or PTX-induced Bcl2 phosphorylation at T69 was also inhibited by NAC treatment (Supplementary information, Figure S5I and S5J). These results indicate that $\mathrm{TNF} \alpha$ - or $\mathrm{PTX}$-induced $\mathrm{Bcl} 2$ regulation by PKM 2 was ROS dependent, suggesting a specific role of PKM2 under oxidative stress.

PKM2-dependent Bcl2 phosphorylation was also investigated in other types of tumor cells. A549 or HT29 cells were depleted of endogenous PKM2 and reconstituted with rPKM2 WT or ILLL, or were depleted of endogenous $\mathrm{Bcl} 2$ and reconstituted with $\mathrm{rBcl} 2 \mathrm{WT}$ or T69A. Consistently, $\mathrm{H}_{2} \mathrm{O}_{2}$ treatment induced more apoptosis in A549 or HT29 cells expressing rPKM2 ILLL or rBcl2 T69A than in the cells expressing rPKM2 WT or rBcl2 WT (Supplementary information, Figure S5K$\mathrm{S} 5 \mathrm{~N})$.

Conditions associated with tumorigenesis (including gliomagenesis) such as hypoxia, matrix detachment, mitochondrial dysfunction and inflammation can all lead

Figure 4 PKM2 phosphorylates Bcl2 at T69. Immunoprecipitation and immunoblotting analyses were performed with the indicated antibodies. Data are representative of at least three independent experiments. (A) U87 cells that stably express shNT or shPKM2 were infected with a lentivirus expressing SFB-Bcl2 and then treated with or without $\mathrm{H}_{2} \mathrm{O}_{2}(0.5 \mathrm{mM}, 1 \mathrm{~h})$. SFB$\mathrm{Bcl} 2$ was pulled down using streptavidin agarose beads. (B) Chaperon assay was performed with recombinant $\mathrm{HSP} 90 \alpha 1$ and GST-PKM2. After reaction, GST-PKM2 was pulled down using glutathione agarose beads. In vitro kinase assays were performed by mixing isomerized GST-PKM2 and His-Bcl2 WT or Bcl2 T69A or Bcl2 S70A. (C) Chaperon assay was performed with recombinant HSP90 1 and GST-PKM2 or GST-PKM1. GST-PKM2 or GST-PKM1 was pulled down using glutathione agarose beads. In vitro kinase assay was performed by mixing His-Bcl2 and isomerized GST-PKM2 or GST-PKM1. (D) Chaperon assay was performed with recombinant HSP90 1 and GST-PKM2. GST-PKM2 was pulled down using glutathione agarose beads. In vitro kinase assays were performed by mixing isomerized GST-PKM2 and His-Bcl2. After reaction, His$\mathrm{Bcl} 2$ was pulled down using Nickel agarose beads and analyzed by mass spectrometry. Mass spectrometric analysis of a tryptic fragment at $\mathrm{m} / \mathrm{z} 182.054$ (mass error was 0.10 p.p.m.) matched to the doubly charged peptide 69-pho-tSPLQTPAAPGAAAGPALSPVPPVVHLTLR-98, suggesting that T69 was phosphorylated. The Sequest score for this match was Xcorr = 2.18; Mascot scores were 27, and expectation value was $5.1 \times 10^{-4}$. The Best score evidence ID was 460 , and site probability was 78.4\%. (E) PKM2-depleted U87 cells that were reconstituted with the expression of rPKM2 WT or ILLL were infected with a lentivirus expressing SFB-Bcl2 and then treated with or without $\mathrm{H}_{2} \mathrm{O}_{2}(0.5 \mathrm{mM}, 1 \mathrm{~h})$. (F, G) U87 cells that stably express SFB$\mathrm{Bcl} 2 \mathrm{WT}$ or T69A were treated with or without $\mathrm{H}_{2} \mathrm{O}_{2}(0.5 \mathrm{mM}, 4 \mathrm{~h})$. The cells were pretreated with MG132 for $0.5 \mathrm{~h}$ before $\mathrm{H}_{2} \mathrm{O}_{2}$ treatment $(\mathbf{F})$. 
A

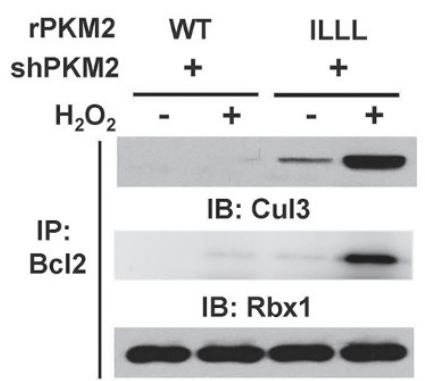

IB: Bcl2

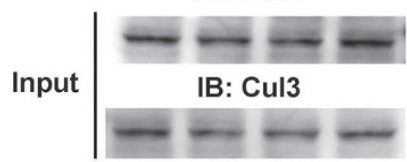

IB: Rbx1

D

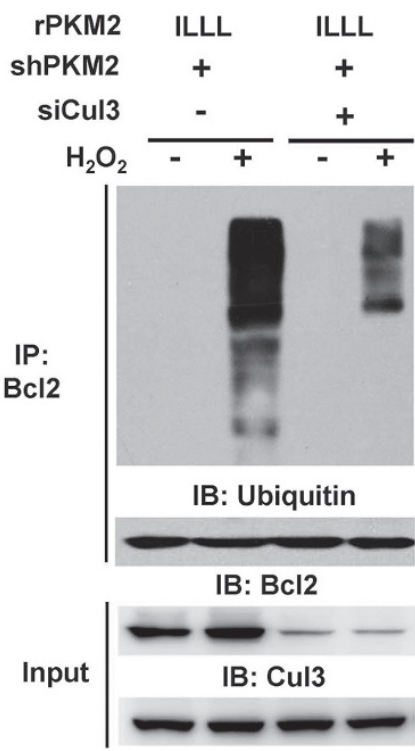

IB: Tubulin
B

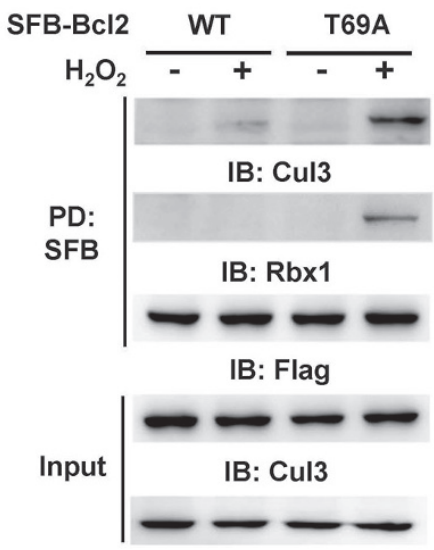

IB: Rbx1

E

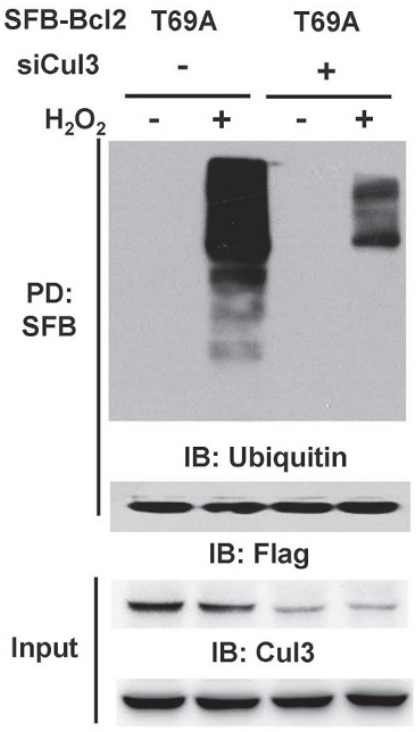

IB: Tubulin
C

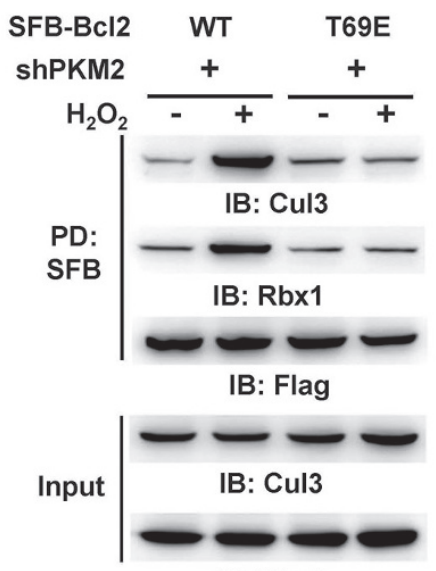

IB: Rbx1

F

G

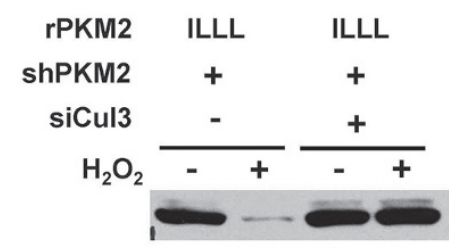

IB: Bcl2

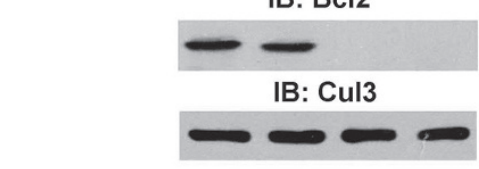

IB: Tubulin

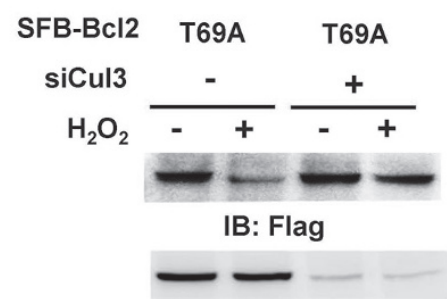

IB: Cul3

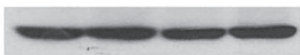

IB: Tubulin

Figure 5 PKM2-dependent Bcl2 T69 phosphorylation prevents the binding of Cul3-based E3 ligase to Bcl2. Immunoprecipitation and immunoblotting analyses were performed with the indicated antibodies. Data are representative of at least three independent experiments. (A) PKM2-depleted U87 cells were reconstituted with the expression of rPKM2 WT or ILLL and then treated with or without $\mathrm{H}_{2} \mathrm{O}_{2}(0.5 \mathrm{mM}, 1 \mathrm{~h})$. (B) U87 cells that stably express SFB-Bcl2 WT or T69A were treated with or without $\mathrm{H}_{2} \mathrm{O}_{2}(0.5 \mathrm{mM}, 1 \mathrm{~h})$. SFB-Bcl2 was pulled down using streptavidin agarose beads. (C) PKM2-depleted U87 cells were infected with a lentivirus expressing SFB-Bcl2 WT or T69E and then treated with or without $\mathrm{H}_{2} \mathrm{O}_{2}(0.5 \mathrm{mM}, 1 \mathrm{~h})$. SFB-Bcl2 was pulled down using streptavidin agarose beads. (D, F) PKM2-depleted U87 cells were reconstituted with the expression of rPKM2 ILLL and then transfected with or without siRNA against CUL3 (siCul3). The cells were treated with or without $\mathrm{H}_{2} \mathrm{O}_{2}$ $(0.5 \mathrm{mM}, 4 \mathrm{~h})$. The cells were pretreated with MG132 for $0.5 \mathrm{~h}$ (D). (E, G) U87 cells that stably express SFB-Bcl2 T69A were transfected with or without siCul3 and then treated with or without $\mathrm{H}_{2} \mathrm{O}_{2}(0.5 \mathrm{mM}, 4 \mathrm{~h})$. The cells were pretreated with MG132 before $\mathrm{H}_{2} \mathrm{O}_{2}$ treatment (E). 
A
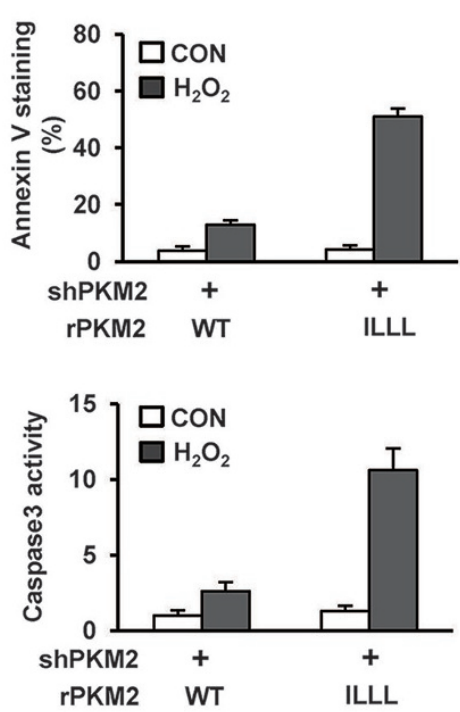

D

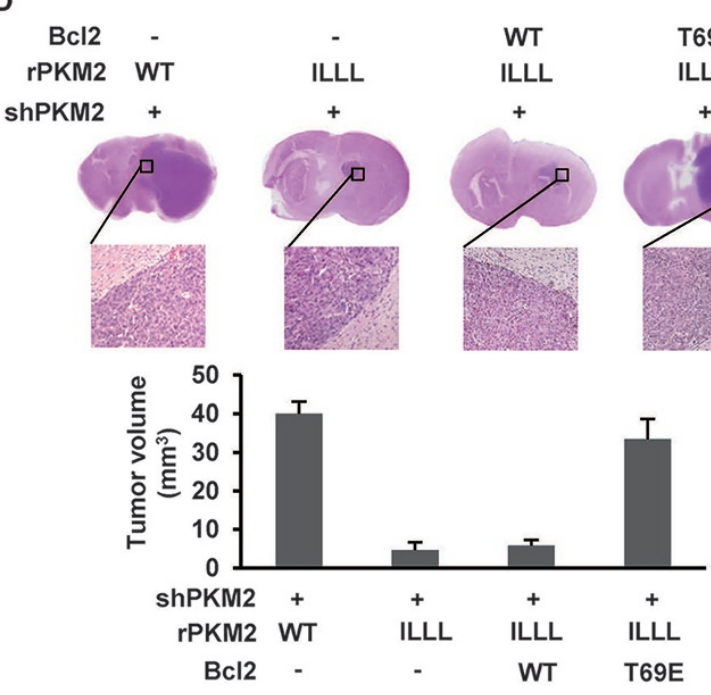

$\mathrm{H}$

G

rBcl2 WT T69A

shBcl2

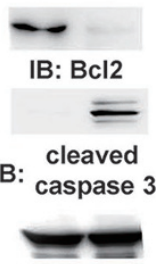

IB: Tubulin
B
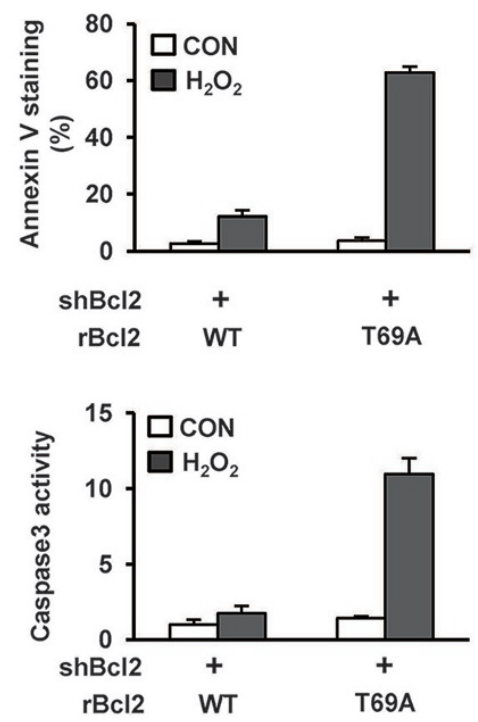

E
C
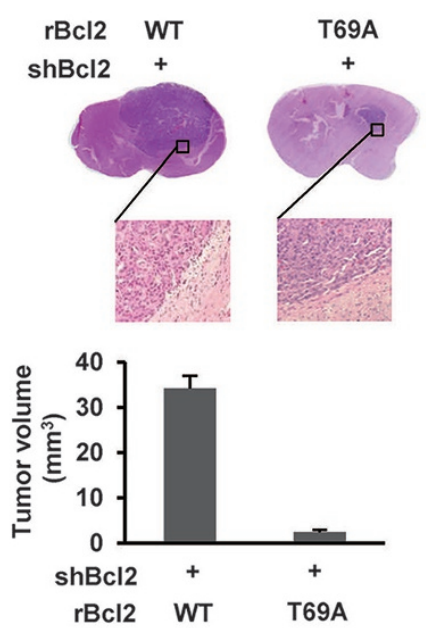

F
- shBcl2+rBcl2 WT

- shBcl2+rBcl2 T69A

- shPKM2+rPKM2 WT

- shPKM2+rPKM2 ILLL

- ᄂ. shPKM2+rPKM2 ILLL+Bcl2 WT

-2. shPKM2+rPKM2 ILLL+Bcl2 T69E

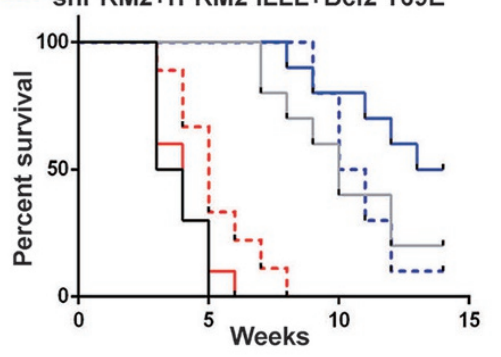

I - Flag peptides

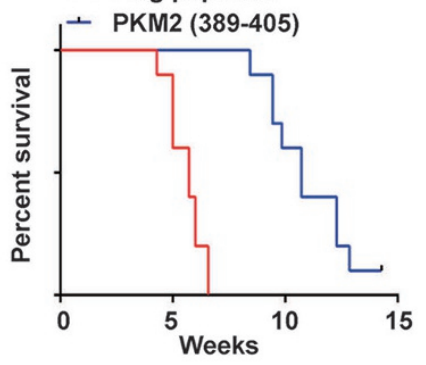

J

Flag peptides + -

PKM2 (389-405) - +

IB: cleaved

B: caspase 3

IB: Tubulin
IB: Bcl2 
to excess production of ROS [32-34]. Therefore, cancer cells are particularly challenged while dealing with oxidative stress $[35,36]$. To evaluate the contribution of PKM2-dependent Bcl2 T69 phosphorylation to brain tumor development, we intracranially injected (1) Bcl2-depleted U87 cells with reconstituted expression of $\mathrm{rBcl} 2$ WT or T69A, (2) PKM2-depleted U87 cells with reconstituted expression of rPKM2 WT or ILLL, or (3) Bcl2 WT- or T69E-expressing U87 cells that were depleted of PKM2 and reconstituted with the expression of rPKM2 ILLL (Supplementary information, Figure S5O) into randomized athymic nude mice. Analyses of hematoxylin and eosin (H\&E) staining and tumor volume showed that cells expressing $\mathrm{rPKM} 2 \mathrm{WT}$ or $\mathrm{rBcl} 2 \mathrm{WT}$ elicited rapid tumorigenesis. In contrast, brain tumor development was markedly dampened by the expression of rPKM2 ILLL or rBcl2 T69A. Notably, rPKM2 ILLL expression-suppressed tumorigenesis was restored by Bcl2 T69E expression, but not Bcl2 WT expression (Figure 6C and 6D). Clear boundaries between tumor cells and normal cells without obvious inflammatory lesions were observed in the brain sections from the mice inoculated with genetically-modified U87 cells (Figure 6C and 6D, middle panel). Kaplan-Meier survival curves showed that mice injected with the cells expressing rBcl2 T69A or rPKM2 ILLL survived longer time than mice injected with the cells expressing rBcl2 WT or rPKM2 WT. Consistently, the expression of Bcl2 T69E, but not Bcl2 WT, could significantly shorten the survival time of the mice inoculated with PKM2 ILLL-reconstituted U87 cells (Figure 6E).

Further, the contribution of PKM2-dependent Bcl2 T69 phosphorylation to gliomagenesis was evaluated using luciferase-labeled U87 cells. Quantification of real-time imaging of tumor growth showed that PKM2-depleted U87 cells expressing rPKM2 WT or Bcl2-depleted cells expressing $\mathrm{rBcl} 2$ WT expanded quickly in mice 15 days after implantation and reached maximum number after 40 days, whereas cells expressing rPKM2 ILLL or rBcl2 T69A failed to do so (Supplementary information, Figure S5P and S5Q). Consistent results were also obtained in mice implanted with (1) PKM2-depleted U251 cells with reconstituted expression of rPKM2 WT or rPKM2 ILLL, and (2) Bcl2-depleted U251 cells with reconstituted expression of $\mathrm{rBcl} 2$ WT or T69A. Kaplan-Meier survival curves showed that mice injected with U251 cells expressing rPKM2 ILLL or rBcl2 T69A had longer survival time than mice injected with U251 cells expressing rPKM2 WT or rBcl2 WT (Supplementary

Figure 6 Phosphorylation of Bcl2 T69 by PKM2 enhances the resistance of tumor cells to oxidative stress and promotes gliomagenesis. Immunoblotting analyses were performed with the indicated antibodies. Data are representative of at least three independent experiments. (A) U87 cells were depleted of endogenous PKM2 and then reconstituted with the expression of rPKM2 WT or ILLL. The cells were treated with or without $\mathrm{H}_{2} \mathrm{O}_{2}(0.5 \mathrm{mM}, 24 \mathrm{~h})$. The cells were harvested for analyses of apoptosis using Annexin $\mathrm{V}$ staining (top panel) or caspase 3 activity (bottom panel). Data represent the means \pm SD of three independent experiments. (B) U87 cells were depleted of endogenous $\mathrm{Bcl} 2$ and then reconstituted with the expression of $\mathrm{rBcl} 2 \mathrm{WT}$ or T69A. The cells were treated with or without $\mathrm{H}_{2} \mathrm{O}_{2}(0.5 \mathrm{mM}, 24 \mathrm{~h})$. The cells were harvested for analyses of apoptosis using Annexin V staining (top panel) or caspase 3 activity (bottom panel). Data represent the means \pm SD of three independent experiments. (C, D) U87 cells were depleted of endogenous $\mathrm{Bcl} 2$ and reconstituted with the expression of $\mathrm{rBcl} 2$ WT or T69A (C). U87 cells were depleted of endogenous PKM2 and reconstituted with expression of rPKM2 WT or ILLL (D). Endogenous PKM2-depleted U87 cells with reconstituted expression of rPKM2 ILLL were further infected with the lentivirus expressing Flag-Bcl2 WT or T69E (D). All genetically-modified U87 cells above were harvested and intracranially injected into randomized athymic nude mice (seven mice per group). After 40 days, the mice were euthanized and tumor growth was examined. H\&E-stained coronal brain sections show representative tumor xenografts (top panel). Representative images of tumor boundaries were presented in middle panel with $200 \times$ magnification. Tumor volumes were measured using length (a) and width $(b)$ and calculated using the equation: $V=a b^{2} / 2$ (bottom panel). Data represent the means $\pm S D$ of seven mice. (E) Kaplan-Meier survival curves for the mice intracranially injected with Bcl2-depleted U87 cells reconstitutively expressing rBcl2 WT or T69A, or PKM2-depleted U87 cells reconstitutively expressing rPKM2 WT or ILLL, or Bcl2 WT- or T69E-expressing $\mathrm{U} 87$ cells that were depleted of PKM2 and reconstituted with the expression of rPKM2 ILLL (10 mice per group). (F, G) Tumor tissues derived from the mice injected with PKM2-depleted U87 cells reconstitutively expressing rPKM2 WT or ILLL (F), or Bcl2-depleted U87 cells reconstitutively expressing rBcl2 WT or T69A (G) were dissected for immunoblotting analyses. (H, J) U87 cells were intracranially injected into randomized athymic nude mice. After 15 days, 7 mice were sacrificed to examine tumor growth; the remaining mice were intratumorally injected with the lentivirus expressing Flag or PKM2 (389-405) peptides, and then sacrificed on day 40. H\&E-stained coronal brain sections show representative tumor xenografts $(\mathbf{H}$, top panel). Representative images of tumor boundaries were presented in middle panel with $200 \times$ magnification. Tumor volumes were calculated $(\mathbf{H}$, bottom panel). Data represent the means \pm SD of 7 mice. Tumor tissues derived from the mice injected with the lentivirus expressing Flag or PKM2 389-405 peptides were dissected for immunoblotting analyses (J). (I) U87 cells were intracranially injected into randomized athymic nude mice. After 15 days, the mice were intracranially injected with the lentivirus expressing Flag or PKM2 (389-405) peptides. Kaplan-Meier survival curves were then plotted. 
information, Figure S5R). Furthermore, immunoblotting analyses of the dissected tumor tissues showed that decreased levels of Bcl2 and increased levels of cleaved caspase 3 were detected in tumor tissues derived from the mice injected with U87 cells expressing rPKM2 ILLL or rBcl2 T69A compared to those in the counterpart tissue derived from the mice injected with U87 cells expressing rPKM2 WT or rBcl2 WT (Figure 6F and 6G). Together, these results demonstrate that $\mathrm{Bcl} 2 \mathrm{~T} 69$ phosphorylation by PKM2 is crucial for brain tumor development.

We next investigated therapeutic effects of PKM2 389-405 peptide on gliomagenesis. U87 cells were intracranially injected into randomized nude mice. After 15 days, the mice bearing relatively small tumors were intratumorally injected with a lentivirus expressing Flag or PKM2 389-405 peptides. On day 40, analyses of H\&E staining and tumor volume showed that expression of PKM2 389-405 peptide, but not Flag peptide, significantly impaired tumor growth (Figure 6H). Kaplan-Meier survival curves showed the mice injected with the lentivirus expressing PKM2 389-405 peptide had much longer survival time than the mice injected with the lentivirus expressing Flag peptides (Figure 6I). Luciferase-labeled U87 cells were also used to evaluate therapeutic potential of PKM2 peptides. As shown in Supplementary information, Figure S5S, luciferase-expressing U87 cells grew into a small tumor 15 days after implantation. On day 40 , the mice injected with the lentivirus expressing PKM2 389-405 peptide bear much smaller tumor than the mice injected with the lentivirus expressing Flag peptides. Consistent results were obtained in mice implanted with U251 cells followed by the injection of the lentivirus expressing Flag peptide or PKM2 389-405 peptide, showing that the expression of PKM2 peptide markedly prolonged mouse survival (Supplementary information, Figure S5T). Immunoblotting analyses of the dissected tumor tissues showed decreased level of Bcl2 and increased level of cleaved caspase 3 in tumor tissues derived from the mice injected with the lentivirus expressing PKM2 389-405 peptide compared to those in the counterpart tissues derived from the mice injected with the lentivirus expressing Flag peptides (Figure 6J). Together, these results demonstrate that PKM2 389-405 peptide could inhibit gliomagenesis effectively, highlighting therapeutic potential of PKM2 peptide as an anti-tumor drug.

Bcl2 pT69 levels positively correlate with levels of conformation-altered PKM2 and grades of glioma malignancy and prognosis

To investigate clinical significance of PKM2-dependent Bcl2 T69 phosphorylation, immunohistochemistry
(IHC) analyses were performed with 40 specimens from human primary GBM patients (World Health Organization (WHO) grade IV) using antibodies of anti-Bcl2, anti-Bcl2 pT69 or anti-PKM2-389, which specifically recognizes PKM2 with conformational change. Specificities of the antibodies used for IHC staining were validated by a blocking peptide/recombinant protein competition assay (Supplementary information, Figure S6). As shown in Figure 7A, Bcl2 protein levels correlated with levels of conformation-altered PKM2 or Bcl2 pT69. Pearson correlation analysis of the staining on a scale of $0-8.0$ showed that these correlations were significant (Figure $7 \mathrm{~B}$ and $7 \mathrm{C})$.

We next examined the correlation of survival of 80 GBM patients, all of whom received standard adjuvant radiotherapy after surgery and the following treatment with an alkylating agent (TMZ in most cases), with levels of Bcl2 pT69 (low, 0-4.5 staining score; high, 4.6-8 staining score) or levels of PKM2 with conformational change (low, 0-3.9 staining score; high, 4.6-8 staining score). Patients whose tumors had low levels of conformation-altered PKM2 (22 cases) had a median survival of 169.58 weeks, whereas those whose tumors had high levels of conformation-altered PKM2 (58 cases) had a significantly lower median survival of 69.33 weeks (Figure 7D). Patients whose tumors had low levels of Bcl2 pT69 (28 cases) had a median survival of 166.66 weeks, whereas those whose tumors had high levels of Bcl2 pT69 (52 cases) had a significantly lower median survival of 59.34 weeks (Figure 7E). Correlations of levels of Bcl2 pT69 and conformation-altered PKM2 with grades of glioma malignancy were also explored. Levels of conformation-altered PKM2 or Bc12 pT69 in samples from patients with low-grade diffuse astrocytoma (WHO grade II; median survival time $>5$ years) were compared with those from patients with high-grade GBM [37]. IHC analyses showed that levels of conformation-altered PKM2 or Bcl2 pT69 in low-grade tumors were significantly lower than those in GBM tumors (Figure 7F and $7 \mathrm{G})$.

Taken together, these results clarify the importance of PKM2-dependent Bcl2 phosphorylation and stabilization in the clinical behavior of human GBM and reveal a relationship between Bcl2 T69 phosphorylation or conformation alteration of PKM2 and clinical glioma malignancy.

\section{Discussion}

PKM2 is upregulated in many human cancers and promotes tumor cell proliferation through multiple mechanisms. Recent studies reported that PKM2 also regulates apoptosis. However, mechanisms underlying such a role 
A

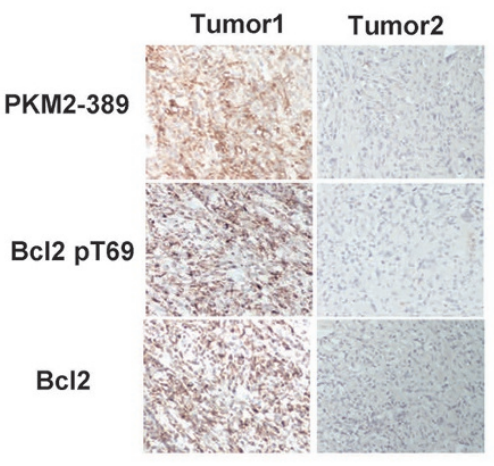

D

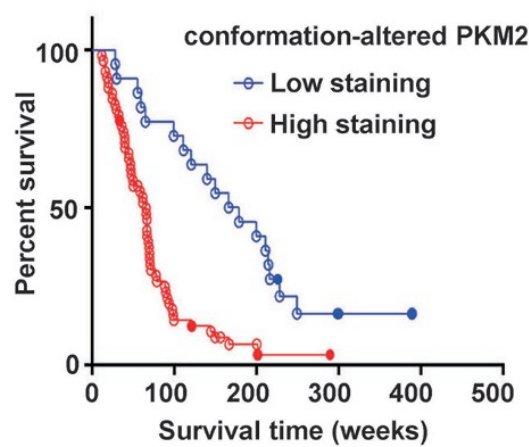

B

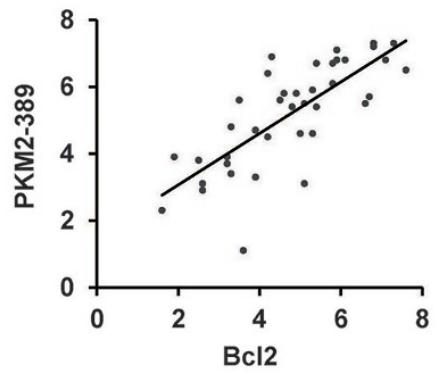

E

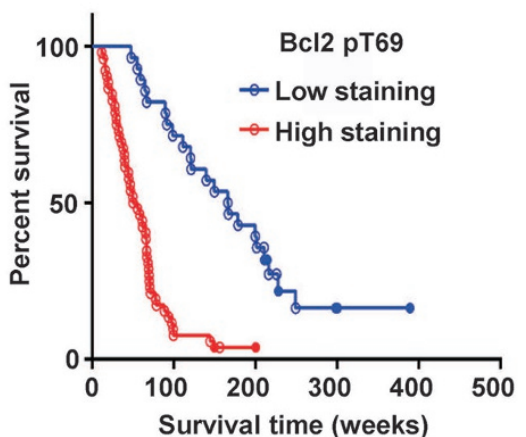

C
G

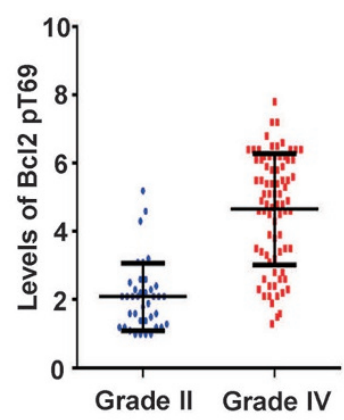

H

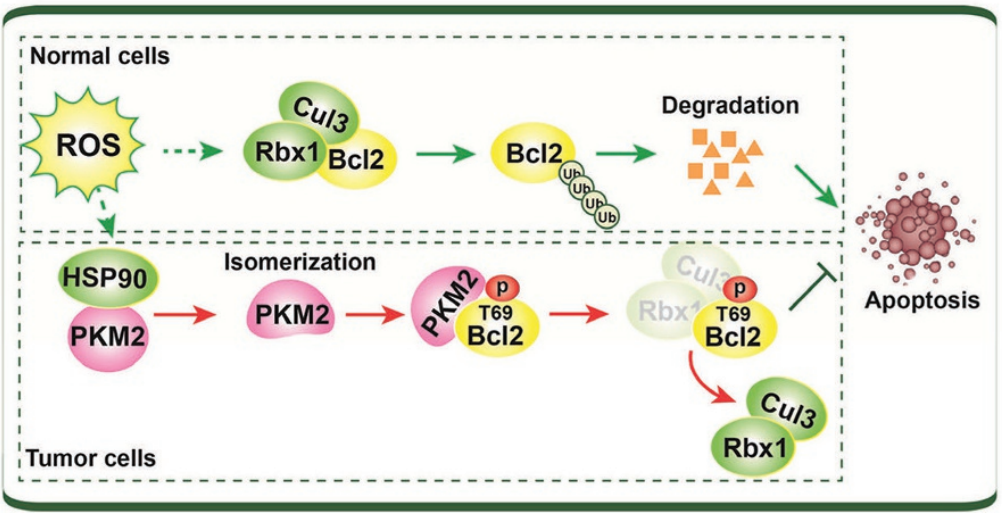

Figure $7 \mathrm{Bcl} 2$ pT69 levels positively correlates with levels of conformation-altered PKM2 and grades of glioma malignancy and prognosis. (A-C) Immunohistochemical analyses of 40 specimens from GBM patients using anti-PKM2-389, anti-Bcl2 pT69 and anti-Bcl2 antibodies were performed. Representative images of two GBM specimens (A). Semi-quantitative scoring (using a scale from 0 to 8 ) was carried out (Pearson product moment correlation test; $\mathbf{B}, r=0.76, P<0.001$; $\mathbf{C}, r=0.81$, $P<0.001)$. (D, E) Survival of 80 patients with low (0-4.5 staining scores, blue curve) versus high (4.6-8 staining scores, red curve) conformation-altered PKM2 levels (low, 22 patients; high, 58 patients) was analyzed (D). Survival of 80 patients with low (0-3.9 staining scores, blue curve) versus high (4.0-8 staining scores, red curve) Bcl2 T69 phosphorylation levels (low, 28 patients; high, 52 patients) was compared (E). Empty circles represent the deceased patients, and filled circles represent the censored (alive at last clinical follow-up) patients. (F, G) Forty diffuse astrocytoma specimens were immunohistochemically stained using PKM2-389 antibody (F) or anti-Bcl2 pT69 antibody (G). Staining scores of the specimens were compared with $40(\mathbf{F})$ or 80 (G) stained GBM specimens (Student's $t$-test, two tailed, $P<0.001)$ ) (H) Schematic representation of the PKM2-mediated Bcl2 stabilization and apoptosis resistance. PKM2 translocates to the mitochondria under oxidative stress, where PKM2 interacts with and phosphorylates Bcl2 at T69. Bcl2 T69 phosphorylation that requires HSP90 1-mediated $\mathrm{PKM} 2$ isomerization prevents the binding of Cul3-based E3 ligase to Bcl2, which stabilizes Bcl2 and therefore inhibits apoptosis and promotes tumorigenesis. 
of PKM2 remains unknown. Here we domonstrate that oxidative stress induces mitochondrial translocation of PKM2. In the mitochondria, PKM2, functioning as a protein kinase, interacts with and phosphorylates $\mathrm{Bcl} 2$ at T69, which disrupts assocation of Cul3-based E3 ligase with $\mathrm{Bcl} 2$, and blocks proteasome-mediated $\mathrm{Bcl} 2$ degradation, thereby inhibiting oxidative stress-induced apoptosis (Figure $7 \mathrm{H}$ ). These results reveal a novel mechanism by which PKM2 regulates apoptosis directly, and highlight the critical role of PKM2 in ROS adaptation of tumor cells. Importantly, levels of Bcl2 T69 phosphorylation and conformation-altered PKM2 correlate with grades of glioma malignancy and prognosis of GBM patient, suggesting that Bcl2 T69 phosphorylation and conformational change of PKM2 could be two biomarkers to predict brain tumor malignancy and prognosis. Furthermore, the finding that PKM2 389-405 peptide disrupts PKM2-Bcl2 interaction, promotes Bcl2 degradation, and hampers brain tumor development points to the potential of this peptide as an anti-cancer drug.

Elevated levels of ROS have been detected in almost all cancer types. Cancer cells can adapt to such stress through multiple mechanisms [38]. Here we demonstrate that PKM2 is involved in such adaptation by inhibiting apoptosis. Our data show that increased level of ROS induced mitochondrial translocation of PKM2. Mitochondrial PKM2 interacts with and phosphorylates Bcl2. PKM2-dependent $\mathrm{Bcl} 2$ phosphorylation prevents $\mathrm{Bcl} 2$ degradation and thereby inhibits ROS-induced apoptosis. In addition to apoptosis inhibition, cancer cells also activate ROS-scavenging systems to cope with increased ROS levels [4]. PKM2 is also involved in this process. Increased intracellular ROS level causes inhibition of glycolytic activity of PKM2 through oxidation of cysteine 358 , which diverts glucose flux into the pentose phosphate pathway, thereby generating sufficient reducing potential for detoxification of ROS [39]. Thus, PKM2 can promote the adaptation of cancer cells to ROS at two levels, suggesting therapeutic potential of targeting PKM2.

PKM2 can be regulated at multiple levels, such as posttranslational modification and subcellular localization. It has been shown in our previous studies that PKM2 is phospohorylated at serine 37 by ERK 1 and 2 upon EGFR activation [12]. PKM2 S37 phosphorylation promotes nuclear translocation of PKM2. In the nucleus, PKM2 interacts with $\beta$-catenin and thereby enhances transactivation activity of $\beta$-catenin, which enhances downstream oncogene expression [13]. Nuclear translocation of PKM2 was also observed by Lv et al. [40], showing that PKM2 K433 acetylation that was mediated by the p300 acetyltransferase prevents the binding of FBP to PKM2 and thereby facilitates nuclear translocation of PKM2 and its switch from a cytoplasmic metabolic enzyme to a nuclear protein kinase. Both studies demonstrated nuclear localization and functions of PKM2. In this study, we showed for the first time that PKM2 translocates to the mitochondria under oxidative stress. Submitochondrial fractionation analyses showed that PKM2 translocated to outermembrane of mitochondria (Supplementary information, Figure S1D). It is likely that PKM2-Bcl2 interaction bridges PKM2 to mitochondrial outermembrane, which remains to be clarified.

Protein kinase activity of PKM2 has been extensively reported. PKM2 phosphorylates Histone H3 or STAT3 in the nucleus and regulates various gene expression $[24,41]$. Bub3 and myosin light chain 2 are also phosphorylated by PKM2 to promote mitosis and cytokinesis in the context of cell cycle $[42,43]$. Moreover, protein kinase activity of PKM2 on Histone H3 can be enhanced by succinyl-5-aminoimidazole-4-carboxamide-1-ribose-5'-phosphate (SAICAR) and over 100 proteins were identified to be phosphorylated by PKM2 [44]. Notably, protein kinase activity of PKM2 was further supported by two recent publications, showing (1) that yeast PKM2 homolog directly phosphorylates histone $\mathrm{H} 3$ at T11 both in vivo and in vitro, and (2) that nuclear PKM2 phosphorylates Stat3 and promotes proinflammatory phenotype of macrophages in coronary artery disease through boosting IL- 6 and IL- $1 \beta$ production $[45,46]$. Together, these studies demonstrate the protein kinase activity of PKM2 in the nucleus. Here we showed that oxidative stress stimulates the protein kinase activity of PKM2 to phosphosrylate $\mathrm{Bcl} 2$ at T69 in the mitochondria, which is essential for the anti-apoptotic function of PKM2.

Roles of $\mathrm{Bcl} 2$ can be regulated by phosphorylation. SLAMF1 deficiency in chronic lymphocytic leukemia (CLL) increased ROS accumulation and induced $\mathrm{Bcl} 2$ phosphorylation, thereby promoting autophagic flux [31]. Flubendazole (a potent inducer of autophagy) activates JNK1, leading to Bcl2 phosphorylation at S70 and subsequent release of Beclin1 from Bcl2-Beclin1 complex for autophagy induction [30]. During mitotic arrest, sustained JNK activation dramatically increases Bcl2 phosphorylation, resulting in the inactivation of anti-apoptotic function of $\mathrm{Bcl} 2$ [47]. Mimicking phosphorylation of the putative mitogen-activated protein (MAP) kinase sites of Bcl2 protein (T56, T74 and S87) abolishes TN$\mathrm{F} \alpha$-induced $\mathrm{Bcl} 2$ degradation and apoptosis [48]. Bcl2 phosphorylation (T69, S70 and S87) confers resistance of CLL cells to the BH3 mimetic ABT-737 by impeding direct binding [49]. Together, these studies elaborated that $\mathrm{Bcl} 2$ phosphorylation is a pivotal mechanism for the regulation of $\mathrm{Bcl} 2$ functions. However, role of the phos- 
phorylation in regulation of $\mathrm{Bcl} 2$ anti-apoptotic function is controversial and upstream kinase that phosphorylates $\mathrm{Bcl} 2$ at $\mathrm{T} 69$ remains elusive. In this study, we revealed that PKM2 phosphorylates Bc12 at T69 in the mitochondria, which prevents the binding of Cul3-based E3 ligase to $\mathrm{Bcl} 2$ and stabilizes Bcl2, thereby inhibiting oxidative stress-induced apoptosis. Notably, Bcl2 T69 phosphorylation by PKM2 has been confirmed by in vitro kinase assay and mass spectrometry analyses.

HSP90 plays a number of important roles, such as assisting folding, intracellular transport, maintenance and degradation of proteins as well as facilitating cell signaling [50]. In cancer cells, it can stabilize some proteins encoded by oncogenes, such as EGFR, PI3K and AKT [51-53]. HSP90 possesses ATPase activity and induces conformational changes in client proteins, thereby causing activation of such proteins [54]. In this study, we demonstrate that HSP90 interacts with and isomerizes PKM2 upon oxidative stress. Isomerized PKM2 is required for $\mathrm{Bcl} 2$ phosphorylation and the inhibition of oxidative stress-induced apoptosis. Our finding identifies PKM2 as a new substrate of HSP90 and uncovers a novel mechanism of HSP90 regulating apoptosis via modulation of PKM2.

Our findings demonstrate that PKM2-dependent Bcl2 phosphorylation and stabilization plays an essential role in resistance of tumor cells to oxidative stress-induced apoptosis and gliomagenesis, which not only reveal a novel mechanism of PKM2-mediated ROS adaptation of tumor cells, but also provide a molecular basis for treating rapid proliferating tumors by interfering with the HSP90-PKM2-Bc12 axis.

\section{Materials and Methods}

\section{Materials}

Rabbit monoclonal antibodies against PKM2, Bcl2 pS70, cytochrome $c$, AKT1, Bcl-xl, Bax, Tom20, MnSOD2 and HSP90 $\alpha 1$, and mouse monoclonal antibodies against $\mathrm{Bcl} 2$ and $\mathrm{Chk} 1$, and polyclonal antibodies against VDAC, Cul3 and Rbx1, and mouse anti-rabbit IgG (Conformation Specific; L27A9) were obtained from Cell Signaling Technology (Danvers, MA, USA). Rabbit polyclonal antibody against Bcl2 pT69 was obtained from Thermo Fisher Scientific. Mouse monoclonal antibody against ubiquitin and GST were purchased from Santa Cruz Biotechnology (Santa Cruz, CA, USA). Rabbit polyclonal antibody recognizing PKM2389 (a customer antibody) was obtained from Signalway Antibody (College Park, MD, USA). Mouse monoclonal antibodies for Flag, His and tubulin, and rabbit polyclonal antibody for TIMM22 were purchased from Sigma (St. Louis, MO, USA). Hygromycin, puromycin, and G418 were purchased from EMD Biosciences (San Diego, CA, USA). PolyJet in vitro DNA transfection reagent was from Signagen Laboratories (Rockville, MD, USA). GelCode Blue Stain Reagent was obtained from Pierce (Rockford, IL, USA).

\section{Cell culture}

U87 and U251 GBM cells and 293T cells were maintained in Dulbecco's modified Eagle's medium (DMEM) supplemented with $10 \%$ fetal bovine serum (FBS) and antibiotics. The protein expression and reconstitution experiments were conducted using the established stable cell lines. Transfection was performed as instructed by manufacturer.

\section{DNA constructs and mutagenesis}

PCR-amplified human PKM2 and $\mathrm{Bcl} 2$ were cloned into pcDNA3.1, pCDH, pCDH-Flag, pCDH-SFB, pCold I, pLOC or pGEX-KG vectors. PCR-amplified human HSP90 $\alpha 1$ was cloned into pCMV-Flag, pCDH-Flag or pGEX-KG. Synthesized $3 \times$ PKM2 389-405 fragments or $3 \times$ Flag fragments were cloned into pCDH-Flag. pCDH-SFB-PKM2 $\triangle 370-388$, pCDH-SFB-PKM2 $\triangle 389-405$, pCDH-SFB-PKM2 406-423, pCDH-SFB-PKM2 4424-435, pCDH-SFB-PKM2 ILLL, pCDH-SFB-PKM2 EERR, pLOC-rPKM2, pLOC-rPKM2 ILLL, pCDH-SFB-Bc12 T69A, pCold I-Bcl2 T69A, pCMV-Flag-HSP90 1 G97D, pCMV-FlagHSP90 $\alpha 1 \Delta$ C, pCMV-Flag-HSP90 $\alpha 1$ MD, pCMV-Flag-HSP90 1 $\Delta \mathrm{N}, \mathrm{pCDH}-\mathrm{Flag}-\mathrm{HSP} 90 \alpha 1, \mathrm{pCDH}-\mathrm{Flag}-\mathrm{HSP} 90 \alpha 1$ 1-293, pCDH-Flag-HSP90 $\alpha 1$ 1-360, pCDH-Flag-HSP90 $\alpha 1$ 1-469, pLOCrHSP901, pCDH-Flag-rHSP90 $\alpha 1$ G97D and pGEX-KG-HSP90 $\alpha 1$ G97D were made using the QuickChange site-directed mutagenesis kit (Stratagene, La Jolla, CA, USA). pLOC-rPKM2 contains nonsense mutations of C1170T, C1173T, T1174C and G1176T. pCDH-Flag-rHSP90 $\alpha 1$ contains nonsense mutations of C2433T, A2434C and G2436T.

The pGIPZ control was generated with the control oligonucleotide 5'-GCTTCTAACACCGGAGGTCTT-3'. pGIPZ PKM2 shRNA was generated with 5'-CATCTACCACTTGCAATTA-3' oligonucleotide targeting exon 10 of the PKM2 transcript. pGIPZ Bcl2 shRNA was generated with 5'-AGGTTCACTAAAGCAGTAG-3' oligonucleotide targeting noncoding region of the $B c l 2$ transcript. pGIPZ HSP90 $\alpha 1$ shRNA was generated with 5'-AGGATCTACAGGATGATCA-3'.

\section{Immunofluorescence analysis}

Cells were fixed and incubated with primary antibodies, Alexa Fluor dye-conjugated secondary antibodies and Hoechst 33342 according to the standard protocols. Cells were examined using a deconvolution microscope (Zeiss, Thornwood, NY, USA) with a $63-\AA \AA$ oil immersion objective. Axio Vision software from Zeiss was used to deconvolute $\mathrm{Z}$-series images.

\section{Subcellular fractionation analyses}

U87 or U251 cells were harvested and washed three times with cold PBS. Cytosolic or mitochondrial fractions were prepared using the Mitochondria/Cytosol Fraction Kit (BioVision).

Mitochondrial subfractionation was performed as described previously [55]. Briefly, isolated mitochondria were resuspended in $10 \mu \mathrm{M} \mathrm{KH}_{2} \mathrm{PO}_{4}$ (pH 7.4) for $20 \mathrm{~min}$ on ice. An equal volume of iso-osmotic solution ( $32 \%$ sucrose, $30 \%$ glycerol, $10 \mathrm{mM} \mathrm{MgCl}$ ) was added and spun at $10000 \times g$ for $10 \mathrm{~min}$ at $4{ }^{\circ} \mathrm{C}$. The supernatant were centrifuged at $15000 \times g$ for $1 \mathrm{~h}$ at $4{ }^{\circ} \mathrm{C}$; the pellet and supernatant contained outer membrane and intermembrane space proteins, respectively. Then, the pellet from the first time centrifuge was resuspended in $10 \mu \mathrm{M} \mathrm{KH}_{2} \mathrm{PO}_{4}$ (pH 7.4) for $20 \mathrm{~min}$ on ice, and iso-osmotic solution was added, followed by centrifuge 
at $15000 \times g$ for $1 \mathrm{~h}$ at $4{ }^{\circ} \mathrm{C}$; the pellet and supernatant contained inner membrane and matrix proteins, respectively.

\section{Apoptosis assay}

U87 or U251 cells were treated with or without $\mathrm{H}_{2} \mathrm{O}_{2}(0.5 \mathrm{mM}$, $24 \mathrm{~h})$. The cells were harvested and stained as described in the protocol of Annexin V-Cy5 Apoptosis Kit (BioVision).

\section{ROS measurement}

Total ROS in tumor tissues were measured using the Oxiselect In Vitro ROS/RNS Assay Kit (Cell Biolabs), following the manufacturer's instructions. Briefly, tumor tissues were homogenized in cold PBS solution $(20 \mathrm{mg} / \mathrm{ml})$ and centrifuged at $10000 \times g$ for 5 min. The supernatant was collected for the assay. The fluorescence (Ex480 nm/Em530 nM) was measured using the Synergy HT Multi-Mode Microplate Reader (Bio-Tek instruments, Inc).

\section{Caspase 3 activity assay}

U87 or U251 cells were treated with or without $\mathrm{H}_{2} \mathrm{O}_{2}(0.5 \mathrm{mM}$, $24 \mathrm{~h})$. The cells were harvested and washed three times with cold PBS. Caspase 3 activity was measured as described in the protocol of the Caspase-3 Activity Assay Kit (Cell Signaling Technology).

\section{Immunoprecipitation and immunoblotting analyses}

Extraction of proteins with a modified buffer from cultured cells was followed by immunoprecipitation and immunoblotting with corresponding antibodies, as described previously [56].

\section{Dot blot assay}

Cell lysate was spotted on a nitrocellulose membrane $(0.2 \mu \mathrm{m}$ pore size) and then dried for several hours at room temperature. Membranes were blocked with 3\% (w/v) nonfat dry milk in PBS for $30 \mathrm{~min}$ at room temperature and probed with the indicated antibodies for $1 \mathrm{~h}$ at room temperature. Membranes were washed 2 times for 3 min with PBS and subsequently incubated with a secondary antibody (HRP-conjugated goat anti-rabbit IgG,) in 3\% (w/ v) nonfat dry milk-PBS for $1 \mathrm{~h}$ at room temperature. Membranes are washed 2 times with PBS. Following the last wash, the membrane is exposed to chemiluminescence detection system (Tanon 5200 Multi) and developed using a Pierce ECL Plus Substrate (Thermo Fisher Scientific).

\section{Purification of recombinant proteins}

Wild-type and mutant GST-PKM2, His-Bc12 and GSTHSP90 $\alpha 1$ were expressed in bacteria and purified as described previously [57]. Non-tagged HSP90 $\alpha 1$ was obtained by thrombin cleavage of GST. The purities of the recombinant proteins were verified by gel staining with Coomassie Blue.

\section{Chaperone assay}

Purified HSP90 $\alpha 1(0.5 \mu \mathrm{g})$ and GST-PKM2 $(0.5 \mu \mathrm{g})$ were incubated at $30{ }^{\circ} \mathrm{C}$ for $30 \mathrm{~min}$ with $2 \mathrm{mM}$ ATP in a total volume of 20 $\mu 1$, containing $40 \mathrm{mM}$ HEPES-KOH (pH 7.5) and $2 \mathrm{mM} \mathrm{MgCl}_{2}$.

\section{Pyruvate kinase assay}

The activity of bacterially purified PKM2 WT $(0.1 \mu \mathrm{g})$ and PKM2 ILLL $(0.1 \mu \mathrm{g})$ toward PEP was measured with a pyruvate kinase assay (BioVision, Mountain View, CA, USA) according to the manufacturer's instruction. Data represent the mean $\pm \mathrm{SD}$ of three independent experiments.

\section{In vitro kinase assay}

The PKM2-mediated kinase reactions were performed as described previously [24]. In brief, the bacterially purified recombinant PKM2 (200 ng) was incubated with Bcl2 (100 ng) with kinase buffer (50 mM Tris- $\mathrm{HCl}, \mathrm{pH} 7.5,100 \mathrm{mM} \mathrm{KCl}, 50 \mathrm{mM}$ $\mathrm{MgCl}_{2}, 1 \mathrm{mM} \mathrm{Na} \mathrm{VO}_{4}, 1 \mathrm{mM}$ DTT, 5\% glycerol, $0.05 \mathrm{mM}$ FBP, $0.5 \mathrm{mM}$ PEP or $5 \mu \mathrm{Ci}^{32} \mathrm{P}$-labeled PEP) in $25 \mu \mathrm{l}$ at $25^{\circ} \mathrm{C}$ for $1 \mathrm{~h}$. The reactions were terminated by the addition of SDS-PAGE loading buffer and heated to $100{ }^{\circ} \mathrm{C}$. The reaction mixtures were then subjected to SDS-PAGE analyses. ${ }^{32} \mathrm{P}$-labeled PEP was prepared as described previously [25]. Briefly, reactions were carried out at $25^{\circ} \mathrm{C}$ for $5 \mathrm{~min}$. Reaction mixtures contained $50 \mathrm{mM}$ HEPES, $\mathrm{pH} 7.5,12.5 \mathrm{mM} \mathrm{KF}, 5 \mathrm{mM} \mathrm{MgCl} 2,1 \mathrm{mM}$ oxaloacetate, 0.5 units $\mathrm{PEPCK} / \mathrm{ml}$ and ${ }^{32} \mathrm{P}$-PEP. The reactions were diluted by 10 -fold with water and applied to Vivapure Q columns (Sartorius) to purify ${ }^{32} \mathrm{P}$-labeled PEP.

\section{Quantitative real-time PCR}

Total RNA was extracted with RNeasy Plus Kit (Qiagen, Valencia, CA, USA). cDNA was prepared by a QuantiTect Reverse Transcription Kit (Qiagen) using oligonucleotide (dT) and random primers. Quantitative real-time PCR analysis was performed under the following conditions: $5 \mathrm{~min}$ at $95{ }^{\circ} \mathrm{C}$ followed by 40 cycles at $95{ }^{\circ} \mathrm{C}$ for $30 \mathrm{~s}, 55^{\circ} \mathrm{C}$ for $40 \mathrm{~s}$, and $72{ }^{\circ} \mathrm{C}$ for 1 min using an ABI Prism 7700 sequence detection system. Data were normalized to expression of a control gene ( $\beta$-actin) for each experiment.

The following primer pairs were used for quantitative real-time PCR: Bcl2, 5'-GGTGGGGTCATGTGTGTGG-3' (forward) and 5'-CGGTTCAGGTACTCAGTCATCC-3' (reverse); $\beta$-actin, 5'-CATGTACGTTGCTATCCAGGC-3' (forward) and 5'-CTCCTTAATGTCACGCACGAT-3' (reverse).

\section{Mass spectrometry analysis}

An in vitro PKM2-phosphorylated recombinant Bcl2 was exhaustively acetylated with acetic anhydride and triethylamine in acetonitrile, evaporated to dryness, then resuspended in $50 \mathrm{mM}$ ammonium bicarbonate buffer containing Rapigest (Waters Corp, MA, USA). The sample was heated to $95{ }^{\circ} \mathrm{C}$ for $10 \mathrm{~min}$ and then allowed to cool; $100 \mathrm{ng}$ of sequencing-grade modified trypsin (Promega, Madison, WI, USA) was added. The digestion proceeded overnight at $37^{\circ} \mathrm{C}$ and was analyzed by LC-MS/MS on an Obitrap-XL mass spectrometer (Thermo Fisher Scientific, Waltham, MA, USA).

Proteins were identified by a database search of the fragment spectra against the National SwissProt protein database (EBI) using Mascot v.2.3 (Matrix Science, London, UK) and Sequest (v.1.20) via Proteome Discoverer v.1.3 (Thermo Fisher Scientific). Phosphopeptide matches were analyzed by using PhosphoRS implemented in Proteome Discoverer and manually curated [58].

\section{Intracranial injection}

We intracranially injected U87 or U251 cells $\left(2 \times 10^{5}\right.$ in $5 \mu \mathrm{l}$ of DMEM per mouse) with depletion of endogenous PKM2 and reconstituted expression of rPKM2 WT or ILLL, or the cells with depletion of endogenous $\mathrm{Bcl} 2$ and reconstituted expression of rBcl2 WT or T69A into randomized 4-week-old female athymic nude mice. Intracranial injections were performed as described in 
a previous publication [59]. Seven mice per group in each experiment were included. Mice injected with U87 cells were sacrificed 40 days after implantation. U87 cells were intracranially injected into randomized athymic nude mice. After 15 days, 7 mice were sacrificed to examine tumor growth; the remaining mice were intracranially injected with the lentivirus-expressing Flag or PKM2 (389-405) peptides, and then sacrificed on day 40 . The brain of each mouse was collected, fixed in $4 \%$ formaldehyde and embedded in paraffin. Tumor formation and phenotype were determined by histologic analysis of H\&E-stained sections. Kaplan-Meier survival curves for the mice intracranially injected with the indicated U87 or U251 cells were performed with ten mice per group. The use of mice was in compliance with ethical regulations and was approved by the institutional review board at the Institute of Biochemistry and Cell Biology.

\section{Bioluminescence imaging with IVIS}

Mice were anaesthetized with isoflurane inhalation, and were subsequently intraperitoneally injected with $100 \mu \mathrm{lof} 7.5 \mathrm{mg} / \mathrm{ml}$ D-luciferin (Xenogen). Bioluminescence imaging with a CCD camera (IVIS, Xenogen) was initiated 10 min after injection with 2 min exposure time. Bioluminescence from the region of interest was defined manually. Background was defined using a region of interest from a mouse that was not given an intraperitoneal injection of D-luciferin. All bioluminescent data were collected and analyzed using IVIS.

\section{Immunohistochemical analysis}

The tissue sections from paraffin-embedded human GBM and astrocytoma specimens were stained with antibodies. We quantitatively scored the tissue sections according to the percentage of positive cells and staining intensity, as previously defined [60]. We rated the intensity of staining on a scale of $0-3: 0$, negative; 1 , weak; 2 , moderate; and 3 , strong. We assigned the following proportion scores: 0 if $0 \%$ of the tumor cells showed positive staining, 1 if $0 \%-1 \%$ of cells were stained, 2 if $2 \%-10 \%$ were stained, 3 if $11 \%-30 \%$ were stained, 4 if $31 \%-70 \%$ were stained, and 5 if $71 \%$ $100 \%$ were stained. We then combined the proportion and intensity scores to obtain a total score (range, $0-8$ ), as described previously [60]. Scores were compared with overall survival, defined as the time from the date of diagnosis to death or last known date of follow-up. All patients received standard adjuvant radiotherapy after surgery, followed by treatment with an alkylating agent (TMZ in most cases). The use of human brain tumor specimens and the database was approved by the Institutional Review Board at Fudan University Shanghai Cancer Center and the Institutional Review Board at First Affiliated Hospital of Wenzhou Medical College. Informed consent was obtained from all patients. Data represent the mean \pm SD of 80 stained GBM specimens and 40 stained astrocytoma specimens.

\section{Statistical analysis}

We determined the significance of differences in the human glioma data using Pearson's correlation test and Student's $t$-test (twotailed). $P<0.05$ was considered to be significant.

\section{Acknowledgments}

This work was supported by the Ministry of Science and Tech- nology of China (2014CB943101) to WY, the National Natural Science Foundation of China (31471324 and 31422034 to WY, and 81402457 to JL), the Strategic Priority Research Program of the Chinese Academy of Sciences (XDB19000000) to WY and the Thousand Talents Plan-Youth to WY.

\section{Author Contributions}

This study was conceived by WY; WY and JL designed the study; JL, RC, XW, PW, YZ and HG performed the experiments; FY, PW, DL and WL provided pathology assistance; CL and RZ provided technical assistance of mass spectrometry analyses; WY wrote the manuscript with comments from all authors.

\section{Competing Financial Interests}

The authors declare no competing financial interests.

\section{References}

1 Reuter S, Gupta SC, Chaturvedi MM, Aggarwal BB. Oxidative stress, inflammation, and cancer: how are they linked? Free Radic Biol Med 2010; 49:1603-1616.

2 Visconti R, Grieco D. New insights on oxidative stress in cancer. Curr Opin Drug Discov Devel 2009; 12:240-245.

3 Waris G, Ahsan H. Reactive oxygen species: role in the development of cancer and various chronic conditions. J Carcinog 2006; 5:14.

4 Trachootham D, Alexandre J, Huang P. Targeting cancer cells by ROS-mediated mechanisms: a radical therapeutic approach? Nat Rev Drug Discov 2009; 8:579-591.

5 Schneider BL, Kulesz-Martin M. Destructive cycles: the role of genomic instability and adaptation in carcinogenesis. Carcinogenesis 2004; 25:2033-2044.

6 Martinez-Sanchez G, Giuliani A. Cellular redox status regulates hypoxia inducible factor-1 activity. Role in tumour development. J Exp Clin Cancer Res 2007; 26:39-50.

7 Chen EI, Hewel J, Krueger JS, et al. Adaptation of energy metabolism in breast cancer brain metastases. Cancer Res 2007; 67:1472-1486.

8 David CJ, Chen M, Assanah M, Canoll P, Manley JL. HnRNP proteins controlled by c-Myc deregulate pyruvate kinase mRNA splicing in cancer. Nature 2010; 463:364-368.

9 Noguchi T, Inoue H, Tanaka T. The M1- and M2-type isozymes of rat pyruvate kinase are produced from the same gene by alternative RNA splicing. J Biol Chem 1986; 261:1380713812.

10 Christofk HR, Vander Heiden MG, Harris MH, et al. The M2 splice isoform of pyruvate kinase is important for cancer metabolism and tumour growth. Nature 2008; 452:230-233.

11 Yang W, Xia Y, Cao Y, et al. EGFR-induced and PKCepsilon monoubiquitylation-dependent NF-B activation upregulates PKM2 expression and promotes tumorigenesis. Mol Cell 2012; 48:771-784.

12 Yang W, Zheng Y, Xia Y, et al. ERK1/2-dependent phosphorylation and nuclear translocation of PKM2 promotes the Warburg effect. Nat Cell Biol 2012; 14:1295-1304.

13 Yang W, Xia Y, Ji H, et al. Nuclear PKM2 regulates $\beta$-catenin transactivation upon EGFR activation. Nature 2011; 480:118122.

14 Luo W, Hu H, Chang R, et al. Pyruvate kinase M2 is a 
PHD3-stimulated coactivator for hypoxia-inducible factor 1 . Cell 2011; 145:732-744.

15 Goldberg MS, Sharp PA. Pyruvate kinase M2-specific siRNA induces apoptosis and tumor regression. J Exp Med 2012; 209:217-224.

16 Morfouace M, Lalier L, Oliver L, et al. Control of glioma cell death and differentiation by PKM2-Oct4 interaction. Cell Death Dis 2014; 5:e1036.

17 Meng MB, Wang HH, Guo WH, et al. Targeting pyruvate kinase M2 contributes to radiosensitivity of non-small cell lung cancer cells in vitro and in vivo. Cancer Lett 2015; 356:985993.

18 Stetak A, Veress R, Ovadi J, Csermely P, Keri G, Ullrich A. Nuclear translocation of the tumor marker pyruvate kinase M2 induces programmed cell death. Cancer Res 2007; 67:1602-1608.

19 Yip KW, Reed JC. Bcl-2 family proteins and cancer. Oncogene 2008; 27:6398-6406.

20 Ling YH, Liebes L, Zou Y, Perez-Soler R. Reactive oxygen species generation and mitochondrial dysfunction in the apoptotic response to Bortezomib, a novel proteasome inhibitor, in human H460 non-small cell lung cancer cells. J Biol Chem 2003; 278:33714-33723.

21 Hildeman DA, Mitchell T, Aronow B, Wojciechowski S, Kappler J, Marrack P. Control of Bcl-2 expression by reactive oxygen species. Proc Natl Acad Sci USA 2003; 100:1503515040.

22 Liu X, Kim CN, Yang J, Jemmerson R, Wang X. Induction of apoptotic program in cell-free extracts: requirement for dATP and cytochrome c. Cell 1996; 86:147-157.

$23 \mathrm{Ku} \mathrm{B}$, Liang C, Jung JU, Oh BH. Evidence that inhibition of BAX activation by BCL-2 involves its tight and preferential interaction with the BH3 domain of BAX. Cell Res 2011; 21:627-641.

24 Yang W, Xia Y, Hawke D, et al. PKM2 phosphorylates histone $\mathrm{H} 3$ and promotes gene transcription and tumorigenesis. Cell 2012; 150:685-696.

25 Mattoo RL, Waygood EB. An enzymatic method for $\left[{ }^{32} \mathrm{P}\right]$ phosphoenolpyruvate synthesis. Anal Biochem 1983; 128:245249.

26 Sato S, Fujita N, Tsuruo T. Modulation of Akt kinase activity by binding to Hsp90. Proc Natl Acad Sci USA 2000; 97:10832-10837.

27 Whitesell L, Lindquist SL. HSP90 and the chaperoning of cancer. Nat Rev Cancer 2005; 5:761-772.

28 Wen Z, Zhong Z, Darnell JE Jr. Maximal activation of transcription by Stat 1 and Stat 3 requires both tyrosine and serine phosphorylation. Cell 1995; 82:241-250.

29 Peng B, Ganapathy S, Shen L, et al. Targeting Bcl-2 stability to sensitize cells harboring oncogenic ras. Oncotarget 2015; 6:22328-22337.

30 Alexandre J, Hu Y, Lu W, Pelicano H, Huang P. Novel action of paclitaxel against cancer cells: bystander effect mediated by reactive oxygen species. Cancer Res 2007; 67:3512-3517.

31 Blaser H, Dostert C, Mak TW, Brenner D. TNF and ROS crosstalk in inflammation. Trends Cell Biol 2016; 26:249-261.

32 Vander Heiden MG, Locasale JW, Swanson KD, et al. Evidence for an alternative glycolytic pathway in rapidly proliferating cells. Science 2010; 329:1492-1499.
33 Yuan ZL, Guan YJ, Wang L, Wei W, Kane AB, Chin YE. Central role of the threonine residue within the $\mathrm{p}+1$ loop of receptor tyrosine kinase in STAT3 constitutive phosphorylation in metastatic cancer cells. Mol Cell Biol 2004; 24:9390-9400.

34 Takeda K, Noguchi K, Shi W, et al. Targeted disruption of the mouse Stat3 gene leads to early embryonic lethality. Proc Natl Acad Sci USA 1997; 94:3801-3804.

35 Levine AJ, Puzio-Kuter AM. The control of the metabolic switch in cancers by oncogenes and tumor suppressor genes. Science 2010; 330:1340-1344.

36 Bensaad K, Cheung EC, Vousden KH. Modulation of intracellular ROS levels by TIGAR controls autophagy. EMBO J 2009; 28:3015-3026.

37 Furnari FB, Fenton T, Bachoo RM, et al. Malignant astrocytic glioma: genetics, biology, and paths to treatment. Genes Dev 2007; 21:2683-2710.

38 Liou GY, Storz P. Reactive oxygen species in cancer. Free Radic Res 2010; 44:479-496.

39 Anastasiou D, Poulogiannis G, Asara JM, et al. Inhibition of pyruvate kinase M2 by reactive oxygen species contributes to cellular antioxidant responses. Science 2011; 334:1278-1283.

40 Lv L, Xu YP, Zhao D, et al. Mitogenic and oncogenic stimulation of K433 acetylation promotes PKM2 protein kinase activity and nuclear localization. Mol Cell 2013; 52:340-352.

41 Gao X, Wang H, Yang JJ, Liu X, Liu ZR. Pyruvate kinase M2 regulates gene transcription by acting as a protein kinase. Mol Cell 2012; 45:598-609.

42 Jiang Y, Li X, Yang W, et al. PKM2 regulates chromosome segregation and mitosis progression of tumor cells. Mol Cell 2014; 53:75-87.

43 Jiang Y, Wang Y, Wang T, et al. PKM2 phosphorylates MLC2 and regulates cytokinesis of tumour cells. Nat Commun 2014; 5:5566.

44 Kim JJ, Lee SB, Park JK, Yoo YD. TNF- $\alpha$-induced ROS production triggering apoptosis is directly linked to Romol and Bcl-X(L). Cell Death Differ 2010; 17:1420-1434.

45 Shirai T, Nazarewicz RR, Wallis BB, et al. The glycolytic enzyme PKM2 bridges metabolic and inflammatory dysfunction in coronary artery disease. J Exp Med 2016; 213:337-354.

46 Li S, Swanson SK, Gogol M, et al. Serine and SAM responsive complex SESAME regulates histone modification crosstalk by sensing cellular metabolism. Mol Cell 2015; 60:408421.

47 Meshkini A, Yazdanparast R. Involvement of oxidative stress in taxol-induced apoptosis in chronic myelogenous leukemia K562 cells. Exp Toxicol Pathol 2012; 64:357-365.

48 Dimmeler S, Breitschopf K, Haendeler J, Zeiher AM. Dephosphorylation targets Bcl-2 for ubiquitin-dependent degradation: a link between the apoptosome and the proteasome pathway. J Exp Med 1999; 189:1815-1822.

49 Song T, Chai G, Liu Y, Yu X, Wang Z, Zhang Z. Bcl-2 phosphorylation confers resistance on chronic lymphocytic leukaemia cells to the BH3 mimetics ABT-737, ABT-263 and ABT-199 by impeding direct binding. Br J Pharmacol 2016; 173:471-483.

50 Jackson SE. Hsp90: structure and function. Top Curr Chem 2013; 328:155-240.

51 Sawai A, Chandarlapaty S, Greulich H, et al. Inhibition of Hsp90 down-regulates mutant epidermal growth factor recep- 
tor (EGFR) expression and sensitizes EGFR mutant tumors to paclitaxel. Cancer Res 2008; 68:589-596.

52 Mohsin SK, Weiss HL, Gutierrez MC, et al. Neoadjuvant trastuzumab induces apoptosis in primary breast cancers. $J$ Clin Oncol 2005; 23:2460-2468.

53 Calderwood SK, Khaleque MA, Sawyer DB, Ciocca DR. Heat shock proteins in cancer: chaperones of tumorigenesis. Trends Biochem Sci 2006; 31:164-172.

54 Bukau B, Weissman J, Horwich A. Molecular chaperones and protein quality control. Cell 2006; 125:443-451.

55 She H, Yang Q, Shepherd K, et al. Direct regulation of complex I by mitochondrial MEF2D is disrupted in a mouse model of Parkinson disease and in human patients. J Clin Invest 2011; 121:930-940.

56 Lu Z, Liu D, Hornia A, Devonish W, Pagano M, Foster DA. Activation of protein kinase $\mathrm{C}$ triggers its ubiquitination and degradation. Mol Cell Biol 1998; 18:839-845.

57 Xia Y, Wang J, Liu TJ, Yung WK, Hunter T, Lu Z. c-Jun downregulation by HDAC3-dependent transcriptional repression promotes osmotic stress-induced cell apoptosis. Mol Cell 2007; 25:219-232.

58 Taus T, Kocher T, Pichler P, et al. Universal and confident phosphorylation site localization using phosphoRS. J Proteome Res 2011; 10:5354-5362.
59 Gomez-Manzano C, Alonso MM, Yung WK, et al. Delta-24 increases the expression and activity of topoisomerase I and enhances the antiglioma effect of irinotecan. Clin Cancer Res 2006; 12:556-562.

60 Ji H, Wang J, Nika H, et al. EGF-induced ERK activation promotes CK2-mediated disassociation of $\alpha$-Catenin from $\beta$-Catenin and transactivation of $\beta$-Catenin. Mol Cell 2009; 36:547-559.

(Supplementary information is linked to the online version of the paper on the Cell Research website.)

cc)(하) $\odot$ This work is licensed under a Creative Commons Attribution-NonCommercial-NoDerivs 4.0 Unported License. The images or other third party material in this article are included in the article's Creative Commons license, unless indicated otherwise in the credit line; if the material is not included under the Creative Commons license, users will need to obtain permission from the license holder to reproduce the material. To view a copy of this license, visit http://creativecommons.org/licenses/by-nc-nd/4.0/

(C) The Author(s) 2017 